\title{
Existence of an Intermediate Metallic Phase at the SDW-CDW Crossover Region in the One-Dimensional Holstein-Hubbard Model at Half-Filling
}

\begin{abstract}
Ashok Chatterjee
School of Physics, University of Hyderabad, Hyderabad 500 046, India

Correspondence should be addressed to Ashok Chatterjee, acsp@uohyd.ernet.in

Received 5 September 2009; Accepted 8 December 2009

Academic Editor: Alexandre Sasha Alexandrov

Copyright ( $) 2010$ Ashok Chatterjee. This is an open access article distributed under the Creative Commons Attribution License, which permits unrestricted use, distribution, and reproduction in any medium, provided the original work is properly cited.

The Holstein-Hubbard model serves as a useful framework to investigate this interplay between the phonon-induced electronelectron attractive interaction and the direct Coulomb repulsion and can afford interesting phase diagrams due to competition among charge-density wave (CDW), spin-density wave (SDW), and superconductivity. However the detailed nature of the CDWSDW transition is still not very well known. It is generally believed that the system undergoes a direct insulator to insulator transition from CDW to SDW with the increase of the on-site Coulomb repulsion for a given strength of the electron-phonon coupling and this is the main bottleneck for the polaronic/bipolaronic mechanism of high-temperature superconductivity. We have recently made an investigation to study the nature of the transition from SDW phase to CDW phase within the framework of a one-dimensional Holstein-Hubbard model at half-filling using a variational method. We find that an intervening metallic phase may exist at the crossover region of the CDW-SDW transition. We have also observed that if the anharmonicity of the phonons is taken into account, this metallic phase widens and the polarons become more mobile, which is a more favorable situation from the point of view of superconductivity. We shall finally show that an improved variational calculation widens the metallic phase and makes the polarons more mobile, which reconfirms the existence of the intermediate metallic phase at the SDW-CDW crossover region.
\end{abstract}

\section{Introduction}

The discovery of high-temperature cuprate superconductors is more than twenty years old [1]; however, the basic mechanism involved for inducing superconductivity in these systems has remained hitherto elusive. Though several mechanisms [2] have been suggested over the last three decades, there has hardly been any consensus on any particular model. One of the potential mechanisms that have been advocated for inducing pairing in cuprates is again the electron-phonon interaction [3-16]. There have also been quite a few experiments which have supported this conjecture [17-27]. According to some of these models, electron-phonon interaction being large enough in the high $T_{c}$ materials, polarons, or bipolarons should be the natural quasiparticles in the normal phase of these systems. However, these materials being strongly correlated systems with narrow bands, it is more convincing to describe the corresponding polarons or bipolarons through the Holstein approach $[15,16,28]$ instead of the usual Frohlich picture [29]. There are several temperature scales in these systems. One is $T_{\mathrm{BCS}}$, which is the transition temperature for the polaronic superconductivity through BCS mechanism [30]. The second temperature scale is $T_{\mathrm{BP}}$, which is the temperature at which two polarons can form a stable local bipolaron which is a static pair as opposed to the dynamic Cooper pair of the usual BCS theory. If $T_{\mathrm{BP}}$ is higher than $T_{\mathrm{BCS}}$, then the normal phase would be a bosonic phase containing bipolarons as the quasiparticles. In this case, there should exist a temperature $T_{\mathrm{BEC}}$, the third temperature scale, which is the Bose-Einstein condensation temperature for the bipolarons. The superconducting phase is then naturally the Bose-Einstein condensate of static bipolarons. The polaronic or bipolaronic mechanism for inducing pairing can explain 
many of the properties of high-temperature cuprates, but there has also been a strong opposition to these theories by some researchers. The polaronic/bipolaronic theories require a strong electron-phonon interaction for the formation of polarons and bipolarons which in the opinion of these researchers is the greatest bottleneck of these theories because at large electron-phonon coupling a system would normally prefer to settle into a charge-density wave (CDW) ground state (GS) which is a peierls insulating phase. On the other hand, if the electron-phonon coupling is not too strong to overcome the Coulomb correlation, the system would prefer to be in a spin-density wave (SDW) GS which is an antiferromagnetic Mott insulating phase. Thus, they argue, that the electron-phonon interaction cannot play any role in high- $T_{c}$ superconductivity. However, a deeper and more careful look suggests that there could be yet another effect arising due to the competition between the phononinduced attractive interaction between electrons and the direct Coulomb repulsion which might lead to some kind of a compromise at the crossover region so much so that the transition from one insulating state to the other may not be direct as normally believed but may go through an intermediate phase and it is this phase which is the subject of our interest.

The most suitable model to study the interplay between the electron-phonon interaction and the Coulomb repulsion in narrow-band systems is the Holstein-Hubbard model. Last three decades have witnessed a flurry of investigations $[15,16,31-43]$ on this model primarily for its potential role in the context of high- $T_{c}$ superconductivity, and also for its intrinsic appeal from the point of view of fundamental physics, for it provides a very useful framework for the investigation of polaronic effects on correlated electrons in narrow-band systems. So far, several studies on the Hubbard-Holstein model have revealed that it can afford interesting phase diagrams due to the competition among charge-density wave (CDW), spin-density wave (SDW), and superconductivity as we change the parameters involved in the system. Recently, Takada and Chatterjee [44] (hereafter referred to as TC) have shown for the first time within the framework of one-dimensional (1D) $\mathrm{HH}$ model at half-filling that there may exist an intervening phase at the CDW-SDW crossover region and, interestingly enough, this phase is metallic. This theoretical observation is of great importance because such a metallic state, if exists, would be just ideal for high- $T_{c}$ superconductivity. TC have shown in a subsequent paper [45] that the presence of phonon anharmonicity makes the polarons even more mobile and also widens the metallic phase favoring superconductivity even more. More recently, Krishna and Chatterjee (KC) [46] have made an improved calculation to test the veracity of the results of TC. The goal was to see whether using a more accurate phonon GS yields results which are more conducive or less conducive for the phenomenon of superconductivity. KC have shown that a better variational analysis in fact broadens the metallic region even further, thus lending more credence to the prediction about the existence of the intermediate metallic phase at the CDW-SDW crossover region made earlier by
TC. In this article we shall present a review of the above scenario.

\section{The Model Hamiltonian}

The Holstein-Hubbard Hamiltonian may be written as

$$
\begin{aligned}
H= & -\sum_{\langle i j\rangle_{\sigma}} t_{i j} c_{i \sigma}^{+} c_{i \sigma}+U \sum_{i} n_{i \uparrow} n_{i \downarrow} \\
& +\omega_{0} \sum_{i} b_{i}^{+} b_{i}+\sum_{i \sigma} g n_{i \sigma}\left(b_{i}+b_{i}^{+}\right),
\end{aligned}
$$

where $c_{i \sigma}^{+}\left(c_{i \sigma}\right)$ is the creation (annihilation) operator for an electron with spin $\sigma$ at the ith lattice site, $n_{i \sigma}=c_{i \sigma}^{+} c_{i \sigma}$ is the electron number operator, $t_{i j}$ is the bare hopping integral, and $U$ is the on-site Coulomb interaction energy. The notation $\langle i j\rangle$ in the hoping term denotes that the summation over $i$ and $j$ has to be carried over nearest neighbors only and we shall write $t_{i j}=t . b_{i}^{+}\left(b_{i}\right)$ is the phonon creation (annihilation) operator at the $i$ th site and $\omega_{0}$ is the dispersionless optical phonon frequency. $g$ is the dispersionless electron-phonon interaction strength which may be written as $g=\sqrt{\alpha} \omega_{0}$, where $\alpha$ is the dimensionless electron-phonon coupling constant. In (1) we have chosen units in such a way that $\hbar=1$. As has been alluded to above, the above Hamiltonian is able to provide the CDW and SDW phases; however, the details of the CDW-SDW transition are not at all clear. The usual belief is that the transition is direct. However, an exact-diagonalization study [41] on the two-site HH model has suggested the appearance of a superconducting phase. As related to this issue, a metalinsulator transition has been predicted in the Holstein model using either the density-matrix renormalization group [42] or a unitary transformation method [43]. Thus, our aim has been to carefully consider the transition region at half-filling by approaching the $\mathrm{HH}$ model from the antiadiabatic region in which $t$ is much smaller than $\omega_{0}$. In order to avoid any error that might enter through the approximations involved in dealing with the Coulomb correlation term, we have investigated the one-dimensional (1D) system so that the exact solution of Lieb and $\mathrm{Wu}$ [47] can be taken advantage of.

\section{Theory}

We shall attempt to solve the above Hamiltonian using a variational approach. The first step is to apply a variabledisplacement (VD) Lang-Firsov (LF) canonical transformation [48-50] with a generator $R_{1}$, defined as

$$
R_{1}=g^{\prime} \sum_{i \sigma} n_{i \sigma}\left(b_{i}^{+}-b_{i}\right),
$$

where $g^{\prime}$ is a variational parameter. We assume that $g^{\prime}$ is of form $g^{\prime}=\omega_{0} \sqrt{\alpha} \eta$. In the conventional LF approach [48], one chooses $\eta=1$ so that $g^{\prime}=g$ and obtains the GS energy by averaging the transformed Hamiltonian with respect to the zero-phonon state which, however, would be a good 
enough approximation for strong $\alpha$ in the antiadiabatic limit. In the weak and intermediate coupling region, however, a lower GS energy can be obtained by optimizing $\eta$. Furthermore, our variable Lang-Firsov transformation (VDLFT) assumes that the phonon coherence coefficient depends linearly on the electron concentration, $n_{i}$. The parameter $\eta$ essentially gives a measure of the lattice deformation; in the strong-coupling limit it approaches unity, but in the intermediate-coupling region, it becomes smaller than that, so as to provide a better total energy by balancing the tendency to localization of an electron by the electronphonon coupling and its itinerant nature due to the hopping motion. Then the transformed Hamiltonian reads

$$
\begin{aligned}
H_{1}= & e^{R} H e^{-R} \\
= & -\alpha \omega_{0} \eta(2-\eta) \sum_{i \sigma} n_{i \sigma}-t \sum_{\langle i j\rangle \sigma} e^{\left(x_{i}-x_{j}\right)} c_{i \sigma}^{+} c_{i \sigma} \\
& +\left[U-2 \alpha \omega_{0} \eta(2-\eta)\right] \sum_{i} n_{i \dagger} n_{i \downarrow}+\omega_{0} \sum_{i} b_{i}^{+} b_{i} \\
& +\sqrt{\alpha} \omega_{0}(1-\eta) \sum_{i \sigma} n_{i \sigma}\left(b_{i}^{+}+b_{i}\right),
\end{aligned}
$$

where the operator $x_{i}$ is defined as $x_{i}=\sqrt{\alpha} \eta\left(b_{i}^{+}-b_{i}\right)$. In constructing a trial wave function $\Psi$ for the ground state in the antiadiabatic region, we consider it as a product of the electronic part $\Phi_{\mathrm{el}}$ and the phonon part $\Phi_{\mathrm{ph}}$, whereby $\Phi_{\text {ph }}$ may include electronic variables, either explicitly or implicitly, but it depends on them only as c-number parameters, not quantum variables, in just the same way as the ion positions are regarded in the electronic wave function in the conventional adiabatic Born-Oppenheimer approximation. Then the energy expectation value $E_{0}$ can be evaluated as

$$
E_{0}=\left\langle\Psi\left|H_{1}\right| \Psi\right\rangle=\left\langle\Phi_{\mathrm{el}}\left|H_{\mathrm{eff}}\right| \Phi_{\mathrm{el}}\right\rangle,
$$

where

$$
H_{\text {eff }}=\left\langle\Phi_{\mathrm{ph}}\left|H_{1}\right| \Phi_{\mathrm{ph}}\right\rangle
$$

is the effective electronic Hamiltonian. Normally for the phonon subsystem, one chooses the zero-phonon state, $\prod_{i}\left|0_{i}\right\rangle$, for the ground state of the electron-phonon composite system. In our treatment, however, we have improved on it by assuming that

$$
\left|\Phi_{\mathrm{ph}}\right\rangle=\prod_{i} e^{\left(-h_{i}^{2} / 2\right)-h_{i} b_{i}^{+}}\left|0_{i}\right\rangle
$$

where $h_{i}$ is another variational parameter and $\left|0_{i}\right\rangle$ is the zerophonon state at the $i$ th site. Note that this state is normalized; $\left\langle\Phi_{\mathrm{ph}} \mid \Phi_{\mathrm{ph}}\right\rangle=1$. If $h_{i}$ is taken to be zero, then it is reduced to the zero-phonon state, but a solution with nonzero $h_{i}$ gives a much lower $E_{0}$, especially for a large positive $U$, as we shall show later. In this paper, we shall be interested only in the half-filled case, that is, we shall consider only one electron per site. Therefore, we assume that all the sites are equivalent; consequently we can assume that $h_{i}=h$, for all $i$, which is consistent with the choice of the $i$ independent $\eta$ in defining $R$. This assumption is also in tune with the form of the electron-phonon interaction taken in the initial Hamiltonian where the electron-phonon interaction coefficient $g$ is same for all sites. Then we can derive $H_{\text {eff }}$ in (5) for the $N$-site system using (6) for $\left|\Phi_{\mathrm{ph}}\right\rangle$. One can immediately see that using the phonon state (6) implies first employing a coherent state transformation on $H_{1}$ with a generator $R_{2}$ given by

$$
R_{2}=\sum_{i}\left[h\left(b_{i}^{+}-b_{i}\right)\right]
$$

and then performing an averaging with respect to the unperturbed zero-phonon state. The two transformations performed so far look together as

$$
R_{12}=\sum_{i \sigma}\left[h+\eta \sqrt{\alpha}\left(n_{i \sigma}-\frac{h}{\sqrt{\alpha}}\right)\right]\left(b_{i}^{+}-b_{i}\right) .
$$

The first transformation corresponds to the antiadiabatic limit $(\eta=1)$ while the second transformation corresponds to the adiabatic limit $(\eta=0)$. Thus both the adiabatic and antiadiabatic regions are considered by taking $0<\eta<1$. The effective electronic Hamiltonian (5) is obtained as

$$
\begin{aligned}
H_{\mathrm{eff}}= & N h^{2} \omega_{0}-[\alpha \eta(2-\eta)+2 \sqrt{\alpha} h(1-\eta)] \omega_{0} \sum_{i \sigma} n_{i \sigma} \\
& -t_{\mathrm{eff}} \sum_{\langle i j\rangle} c_{i \sigma}^{+} c_{i \sigma}+U_{\mathrm{eff}} \sum_{i} c_{i \sigma}^{+} c_{i \sigma},
\end{aligned}
$$

where $t_{\text {eff }}$ is the renormalized hopping integral, given by

$$
t_{\mathrm{eff}}=t e^{-\alpha \eta^{2}},
$$

and $U_{\text {eff }}$ is the effective on-site Coulomb interaction, expressed as

$$
U_{\text {eff }}=\mathrm{U}-2 \alpha \omega_{0} \eta(2-\eta)=\tilde{U}+2 \alpha \omega_{0}(1-\eta)^{2},
$$

where $\tilde{U}=U-2 \alpha \omega_{0}$. One can see that the effective hopping parameter $t_{\text {eff }}$ is reduced by the factor $e^{-\alpha \eta^{2}}$, called the Holstein reduced factor which leads to the phenomenon of band narrowing due to polaron formation. The band reduction factor is maximum for $\eta=1$. One may also note that both $t_{\text {eff }}$ and $U_{\text {eff }}$ are independent of $h$, reflecting the fact that the phonon background represented in $\left|\Phi_{\mathrm{ph}}\right\rangle$ is independent of the electron motion in contrast with the situation described by the operator $R$ in (2). Furthermore if $\eta$ is not equal to unity, then $U_{\text {eff }}$ is larger than $\tilde{U}$ due to the retardation effect of phonons on the phonon-mediated attraction.

In one dimension, the effective Hubbard Hamiltonian (7) can be solved exactly using the Bethe ansatz method as was shown by Lieb and Wu. The solution of Lieb and $\mathrm{Wu}[47]$ is, however, valid for $U_{\text {eff }}>0$. We have performed the following transformations [44]:

$$
c_{i \uparrow} \longrightarrow c_{i \uparrow}, \quad c_{i \downarrow} \longrightarrow(-1)^{i} c_{i \downarrow}^{+},
$$




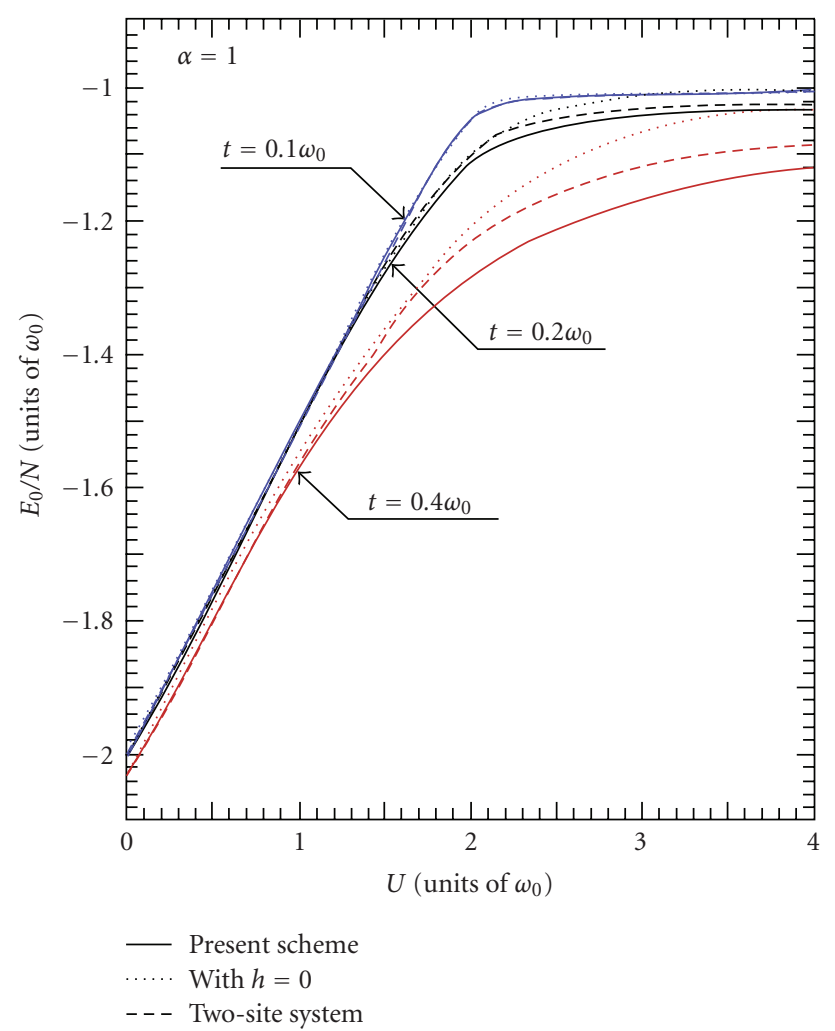

FIGURE 1: (Color online) Ground-state energy per site as a function of $U$ for $\alpha=1$ and three cases of $t / \omega_{0}=0.1,0.2$, and 0.4 .

to obtain results which will be valid for both $U_{\text {eff }}>0$ and $U_{\text {eff }}<0$. The energy per site is obtained as

$$
\begin{aligned}
\epsilon_{0}= & \frac{E_{0}}{N}=-\alpha \omega_{0}+[h-\sqrt{\alpha}(1-\eta)]^{2} \omega_{0} \\
& +\frac{U_{\text {eff }}-\left|U_{\text {eff }}\right|}{4}-4 t_{\text {eff }} \int_{0}^{\infty} \frac{d z}{z} \frac{J_{0}(z) J_{1}(z)}{\left[1+\exp \left(z\left|U_{\text {eff }}\right| / 2 t_{\text {eff }}\right)\right.},
\end{aligned}
$$

where $J_{0}(z)$ and $J_{1}(z)$ are the Bessel functions of zeroth order and first order, respectively. One can immediately see that the variational parameter " $h$ " can be analytically obtained. Namely,

$$
\frac{d \epsilon_{0}}{d h}=0
$$

yields $h=\sqrt{\alpha}(1-\eta)$.

\section{Numerical Results and Discussion}

Minimization with respect to $\eta$ has to be done numerically. The energy per site as function of $U$ for $\alpha=1$ is shown in Figure 1.

In the antiadiabatic limiting region, for example, in the case of $t=0.1 \omega_{0}$, the effect of the parameter $h$ is not so significant, as can be seen by comparing the present result (the solid curve) with the one obtained by choosing $h=0$ (the dotted curve). This is because in this case the optimized value of $\eta$ is pretty close to 1 . The present results for the energy $\epsilon_{0}$ are also in good agreement with the energy of the corresponding two-site system obtained by Takada [41] by using exact diagonalization. This implies that physics of the present system in this region can be essentially captured by the two-site problem. It is evident that at the crossover region there is a rounding-off in the energy. It may also be pointed out that $U_{\text {eff }}$ changes sign in the crossover region. Had the change in the energy been sharp at the crossover region, one would have expected a direct transition from the CDW to the SDW phase. But the rounding off in the energy suggests that there may be an intermediate state at the cross-over region. As $t$ increases, the rounding-off region also increases. Thus adiabaticity seems to favor the intermediate state.

In Figure 2(a), we plot $t_{\text {eff }}$ as a function of $U$ for various values $\alpha$ and for $t=0.2 \omega_{0}$. This is essentially equivalent to plotting the antiadiabaticity parameter $\eta$. A careful observation shows that the curves have some interesting structures. To decipher them, we plot in Figure 2(b) the derivative of $t_{\text {eff }}$ with respect to $U$, that is, $d t_{\text {eff }} / d U$ as a function of $U$. One can immediately see that there are peaks on both sides of the cross-over point defined by $U_{\text {eff }}=0$ which is roughly given by $U=2 \alpha \omega_{0}$. The peak structure becomes more prominent as the electron-phonon coupling constant is increased. As $\alpha$ decreases, the peaks reduce in height and eventually it becomes rather difficult to clearly identify the transition points if $\alpha \omega_{0} \sim 1$. For values of $U$ less than those at the first peak, $t_{\text {eff }}$ is very small which means that $\eta$ is close to 1 . In this case one would expect the formation of massive localized bipolarons or in other words a CDW phase. For values of $U$ larger than the second transition, $\eta$ approaches zero, and thus $t_{\text {eff }}$ tends to the bare hopping integral $t$. This does not, however, mean that the electrons become free. Rather, the system essentially reduces to the original Hubbard model describing the antiferromagnetic insulating phase. Between the two transition points, we observe that $t_{\text {eff }}$ and $U_{\text {eff }}$ obey the condition $t_{\text {eff }} / U_{\text {eff }} \gg 0.25$, which seems to be a sign of a metallic behavior of a system of many free polarons.

In Figure 3, we have plotted the CDW-SDW phase diagram in $(\alpha, U)$ plane. The boundaries of the phase diagram have been obtained from the maxima of the $d t_{\text {eff }} / d U$ versus $U$ curve. We find it difficult to draw the phase diagram for small $\alpha$ or small $U$. However it seems that for $\alpha=0$ and $U<0$, that is, attractive on-site interaction, the normal state is unstable against superconducting transition. We have already argued that the region along increasing $U$ beyond the second peak of $d t_{\text {eff }} / d U$ corresponds to the SDW phase while that along decreasing $U$ from the first peak corresponds to the CDW phase. The region in between the CDW and SDW phases corresponds to $t_{\text {eff }} / U_{\text {eff }} \gg 0.25$ and is, therefore, a metallic phase.

To obtain another evidence for the existence of the intermediate metallic state, we have calculated the average local spin moment per site which is given by

$$
L_{0}=\frac{\sum_{i}\left[\left\langle S_{i}^{2}\right\rangle\right]}{N}=\sum_{i}\left[\left\langle S_{i}^{2}\right\rangle\right]=\frac{N\left[3-6 \sum_{i}\left\langle n_{i \uparrow} n_{i \downarrow}\right\rangle\right]}{4}
$$




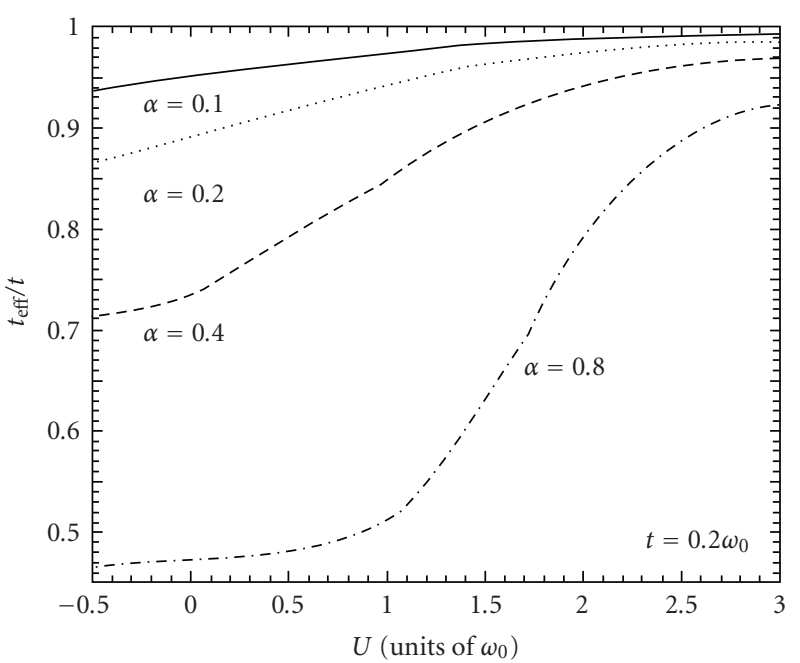

(a)

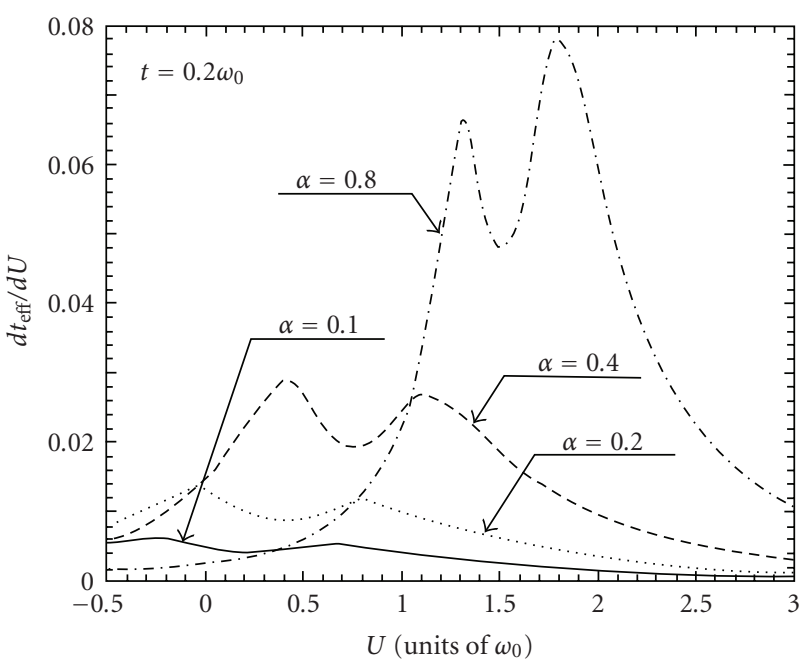

(b)

FIgURE 2: Renormalized hopping integral $t_{\text {eff }}$ and its derivative as a function of $U$ for various values of $\alpha$ at $t=0.2 \omega_{0}$.

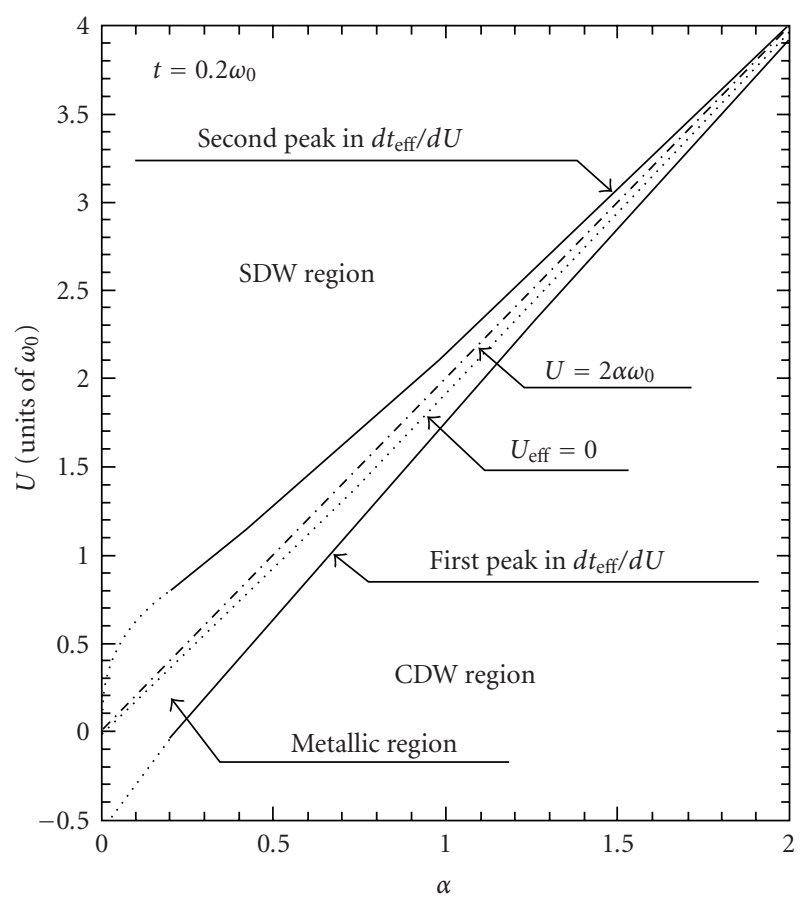

Figure 3: Phase diagram in $(\alpha, U)$ plane determined by the peaks in $d t_{\text {eff }} / d U$. The curve corresponding to $U_{\text {eff }}=0$ is also given by the dotted curve.

which by using (13) can be written as

$$
L_{0}=\frac{\left[3-\left(6 d \epsilon_{0} / d U\right)\right]}{4} .
$$

For totally uncorrelated motion of electrons as in the case of noninteracting electrons, one can easily obtain from (7) the value of $d \epsilon_{0} / d U$ which then yields

$$
L_{0}=\frac{3}{8}=0.375
$$

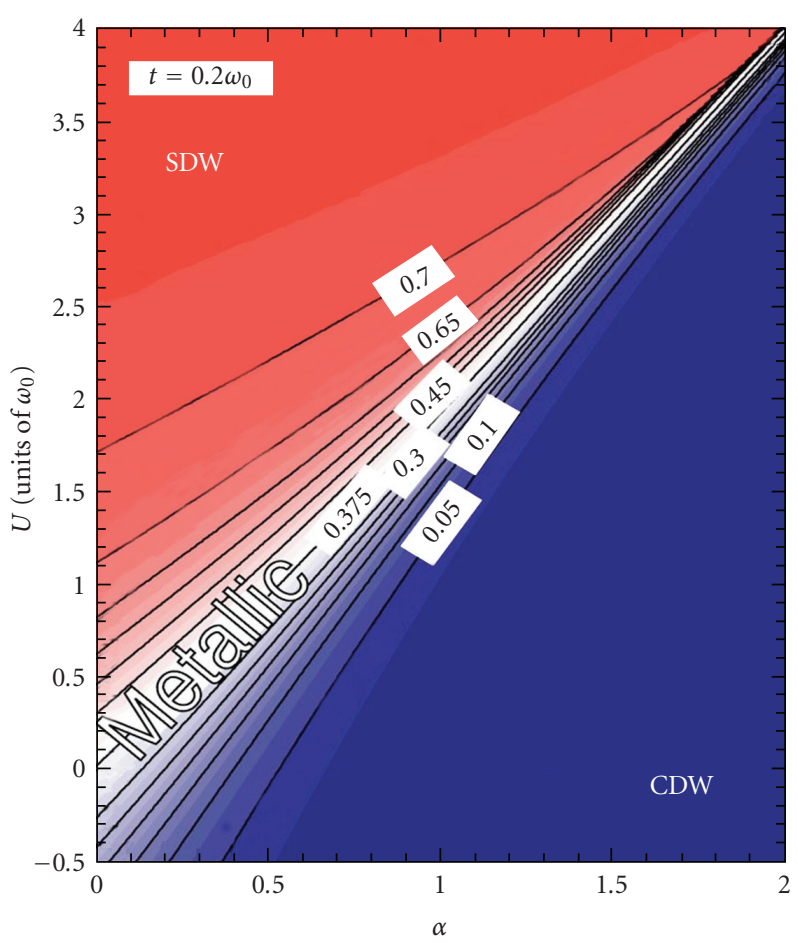

Figure 4: Contour plots of the local moment $L_{0}$ in $(\alpha, U)$ plane.

Using (17) and (13), one can obtain contour plots for $(\alpha, U)$ for different values of $L_{0}$. In Figure 4 we show these plots. It is evident that $L_{0}=0.375$ lies in the same region where $t_{\text {eff }} / U_{\text {eff }} \gg 0.25$, thus confirming the existence of a metallic phase at the cross-over region of the CDW and SDW phases.

\section{Effect of Anharmonic Phonons}

For the sake of simplicity, we have considered the phonons to be harmonic in [44] and in Sections 2-4, while in reality 


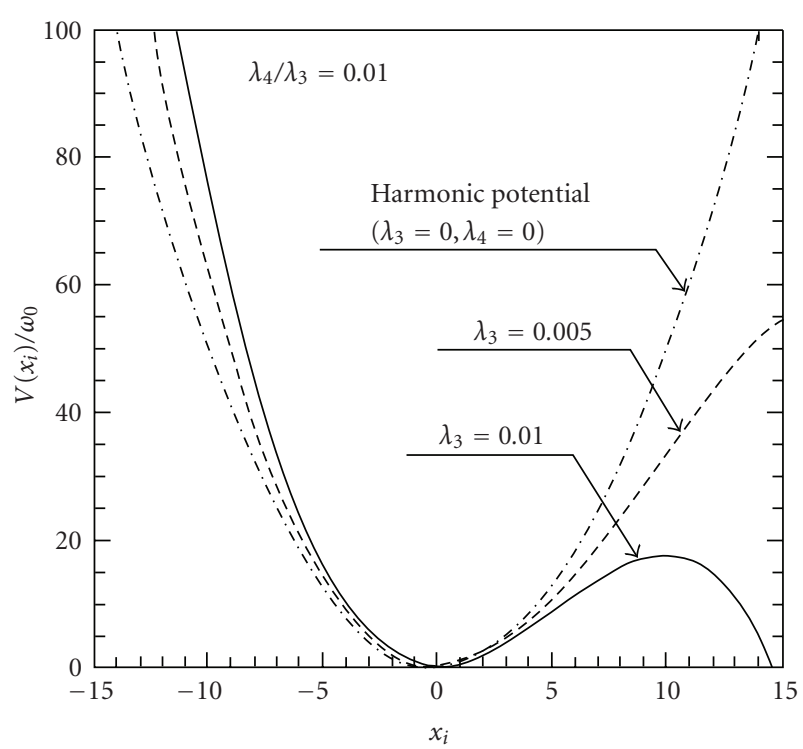

FIGURE 5: Anharmonic potential in units of $\omega_{0}$ as a function of the one-dimensional lattice displacement $x_{i}$ for $\lambda_{3}=0.005$ and 0.01 with $\lambda_{4} / \lambda_{3}=0.01$.

the ion-ion potential in a solid should also contain anharmonic terms giving rise to phonon-phonon interactions, and hence a finite lifetime for phonons. Those phononphonon interaction terms are absolutely necessary to explain certain thermal properties like thermal expansion, but their effects on the electronic properties are normally very small, and therefore they are generally neglected. However, several investigations have shown that phonon anharmonicity can produce some profound effects on the electronic properties as well, particularly in the study of high- $T_{c}$ superconductivity [24-27, 51-53]. In this context, anharmonic vibration of the apex oxygen atoms in cuprates has attracted much attention and its effects have been studied in terms of various models for the anharmonic phonons such as the double-well potential model [54-56] and the Hubbard model coupled to a local two-level system [57]. Since it can mimic a double-well potential with a proper choice of the adjustable parameters, the local Gaussian potential model [58] has also been analyzed. Furthermore, the competition between superconductivity and CDW has been studied in the Holstein-Hubbard model with the quartic anharmonic contribution to the phonon potential energy [59]. One of the features that are common to all these investigations is that the anharmonic potential $V\left(x_{i}\right)$ with the lattice displacement $x_{i}$ is symmetric with respect to the inversion of $x_{i}$ or $x_{i} \rightarrow x_{i}$. Such a symmetric $V\left(x_{i}\right)$, however, would never give rise to thermal expansion of lattice. Chatterjee and collaborators $[60,61]$ have given an appropriate form for $V\left(x_{i}\right)$, which quantitatively explains the thermal expansion behavior of actual materials. It is found that the most dominant anharmonic effect of the lattice potential would come from the terms that are cubic and quartic in the lattice displacement. Indeed, it has recently been shown that the thermal expansion data for both $\mathrm{YBaCuO}$ and $\mathrm{Bi}-$ 2223 superconductors can be explained quite satisfactorily by using $V\left(x_{i}\right)$ consisting of cubic and quartic terms $[62,63]$. This model also predicts the values of the Debye temperature which for both materials compare quite impressively with the values quoted in the literature. This imparts a fair amount of confidence in this model. Since we have come to know a form of the anharmonic lattice potential which is physically motivated and also supported by experiment, it would be interesting to investigate quantitatively how those cubic and quartic anharmonic phonons will modify the polaronic properties in general and the nature of the CDW-SDW transition in particular. We adopt the same variational method as the one introduced in the above Holstein-Hubbard model so that we can make a direct comparison between harmonic and anharmonic models and at the same time restrict ourselves to the one-dimensional problem so that the exact solution of Lieb and $\mathrm{Wu}$ [47] can be used. The present calculation has led us to obtain an important observation that the main effect of the phonon anharmonicities is to effectively shift the on-site Coulomb repulsion $U$ with the direction of the shift depending on the sign of the electron-phonon coupling constant $g$. With the use of the realistic magnitudes for the anharmonicities, we find that the overall features of the anharmonic system are basically similar to those in the harmonic one for positive $g$, while they may become radically different for negative $g$. In the latter case, in particular, a first-order phase transition can be seen, which constitutes an unexpected and very interesting theoretical observation.

The one-dimensional (1D) Hubbard-Holstein model with cubic and quartic anharmonic phonons may be described by the Hamiltonian $H$, given by

$$
\begin{aligned}
H & =H_{e}+H_{\mathrm{ph}}+H_{e-p h}, \text { with } \\
H_{e} & =-t \sum_{\langle i j\rangle_{\sigma}} c_{i \sigma}^{+} c_{j \sigma}+U \sum_{i} n_{i \uparrow} n_{i \downarrow}, \\
H_{\mathrm{ph}} & =\omega_{0} \sum_{i} b_{i}^{+} b_{i}-\omega_{0} \sum_{k=3}^{4} \lambda_{k} \sum_{i}\left(b_{i}^{+}+b_{i}\right)^{k}, \\
H_{e-\mathrm{ph}} & =g \sum_{i \sigma} n_{i \sigma}\left(b_{i}^{+}+b_{i}\right),
\end{aligned}
$$

where $H_{\text {ph }}$ contains the anharmonic phonon terms and rest of the terms are same as in (1).

In terms of the local displacement of an ion at the ith site,

$$
x_{i}\left[\equiv \frac{\left(b_{i}+b_{i}^{+}\right)}{\sqrt{2}}\right]
$$

$H_{\mathrm{ph}}$ in (18) can be derived from the local potential $V\left(x_{i}\right)$ given by

$$
V\left(x_{i}\right)=\omega_{0}\left(\frac{1}{2} x_{i}^{2}-2 \sqrt{2} \lambda_{3} x_{i}^{3}-4 \lambda_{4} x_{i}^{4}\right)
$$


The parameters $\lambda_{3}$ and $\lambda_{4}$ should be positive as required by the consideration of thermal expansion $[60,61,64]$. Quantitatively, their magnitudes can be evaluated by analyzing experiment on thermal expansion of crystals to find that

$$
\lambda_{3} \approx 0.001-0.01, \quad \frac{\lambda_{4}}{\lambda_{3}}=0.001-0.01 .
$$

In Figure 5, $V\left(x_{i}\right)$ is plotted as a function of $x_{i}$ (the dimensionless lattice displacement in some suitable unit) for $\lambda_{3}=0$ (the harmonic case), $0: 005$, and $0: 01$ with $\lambda_{4} / \lambda_{3}=0.01$. It should be noted that, even though it is very small, a positive value of $\lambda_{4}$ eventually leads to a situation where $V\left(x_{i}\right)$ becomes negative for sufficiently large $\left|x_{i}\right|$. However, we shall not be interested in such large variations in $x_{i}$ here; we shall rather look for local minima of the total energy around $x_{i}=0$. As long as both $\lambda_{3}$ and $\lambda_{4}$ are small enough, such local minima are always expected to exist. This restriction to small variations in $x_{i}$ is consistent with the choice of a linear coupling between the lattice displacement and the electron charge in the Hamiltonian. We follow the same variational approach to solve (18) as the one presented in order to solve (1). The energy per site is obtained as

$$
\begin{aligned}
\epsilon_{0}= & \frac{E_{\mathrm{ph}}}{N}+e_{\text {eff }}+\frac{U_{\text {eff }}-\left|U_{\text {eff }}\right|}{4} \\
& -4 t_{\text {eff }} \int_{0}^{\infty} \frac{d z}{z} \frac{J_{0}(z) J_{1}(z)}{\left[1+\exp \left(z\left|U_{\text {eff }}\right| / 2 t_{\text {eff }}\right)\right]},
\end{aligned}
$$

where, as before, $J_{0}$ and $J_{1}$ are, respectively, the zeroth-order and the first-order Bessel functions, $E_{\mathrm{ph}} / N$ is the virtually excited phonon energy given by

$$
\frac{E_{\mathrm{ph}}}{N}=\left[h^{2}+2 \lambda_{3} h(4 h+3)-\lambda_{4}\left(16 h^{2}+24 h^{2}+3\right)\right] \omega_{0},
$$

$e_{\text {eff }}$ is the effective polaron energy given by

$$
\begin{aligned}
e_{\mathrm{eff}}= & -\frac{g^{2}}{\omega_{0}}-h^{2} \omega_{0}+\left[(1-\eta) \frac{g}{\omega_{0}}-h\right]^{2} \omega_{0} \\
& +\lambda_{3} \tilde{\eta}\left[\tilde{\eta}^{2}+6 h \tilde{\eta}+3\left(4 h^{2}+1\right)\right] \omega_{0} \\
& -\lambda_{4} \tilde{\eta}\left[\tilde{\eta}^{3}+8 h \tilde{\eta}^{2}+6\left(4 h^{2}+1\right) \tilde{\eta}+8 \beta\left(4 h^{2}+3\right)\right] \omega_{0}
\end{aligned}
$$

with

$$
\tilde{\eta} \equiv 2 \eta \frac{g}{\omega_{0}},
$$

$t_{\text {eff }}$ is the effective hopping integral given by

$$
t_{\text {eff }}=t \exp \left(-\frac{\tilde{\eta}^{2}}{4}\right)
$$

and the effective on-site Coulomb energy $U_{\text {eff }}$ is given by

$$
\begin{aligned}
U_{\text {eff }}= & U-2 \frac{g^{2}}{\omega_{0}}+2(1-\eta)^{2} \frac{g^{2}}{\omega_{0}}+6 \lambda_{3} \tilde{\eta}^{2}(\tilde{\eta}+2 h) \omega_{0} \\
& -2 \lambda_{4} \tilde{\eta}^{2}\left(7 \tilde{\eta}^{2}+24 h \tilde{\eta}+24 h^{2}+6\right) \omega_{0} .
\end{aligned}
$$

$L_{0}$ is again given by a similar expression as (16). Equation (22) is now minimized with respect to $\eta$ and $h$. The average lattice displacement is obtained as

$$
\left\langle x_{i}\right\rangle=-\sqrt{2}\left(h+\eta \frac{g}{\omega_{0}}\right) .
$$

The variations of $\left\langle x_{i}\right\rangle, t_{\text {eff }}, U_{\text {eff, }}$, and $L_{0}$ as function of $g$ are shown in Figure 6. Though in the absence of the cubic anharmonic term the sign of $g$ is of little physical relevance; in its presence, however, the sign of $g$ becomes quite important. Usually $g$ is taken as a positive quantity and is expressed as $g=\sqrt{\alpha} \omega_{0}$, with $\alpha$ as the dimensionless electron-phonon coupling constant. With this $g$, we would expect that $\left\langle x_{i}\right\rangle$ will not be so much different from its value in the harmonic model, namely, $-\sqrt{2 \alpha}$, as long as both $\lambda_{3}$ and $\lambda_{4}$ are small. This can be easily seen by substituting the harmonic value of $h$ in (28). This implies that $\left\langle x_{i}\right\rangle$ must be negative. According to Figure 5, the potential $V\left(x_{i}\right)$ increases from the corresponding value in the harmonic model for negative $x_{i}$ due to the cubic term, indicating that the cubic anharmonicity increases the total energy. In fact, for positive $g$, the cubic anharmonicity increases the values of both $E_{\mathrm{ph}}$ and $e_{\text {eff, }}$ as can be seen from (23) and (24), respectively. Even more importantly, it shifts $U_{\text {eff }}$ in (27) to the positive side, showing that this anharmonicity gives rise to a repulsive contribution to the effective electron-electron interaction. On the other hand, the quartic anharmonicity has a totally opposite effect on those physical quantities. Thus we may conclude that the effect of anharmonicity on the polaron motion depends on the order of the anharmonicity; the cubic one enhances the effect of $U$, while the quartic one suppresses it. These competing effects of anharmonicity can be well captured in the Holstein-Hubbard model in which the (harmonic) phonon-mediated attractive interaction and the direct Coulomb repulsion are treated on the same footing. For this reason, the Hubbard-Holstein model is quite relevant in clarifying the physics of the cubic and quartic anharmonicities. For negative g given by $-\sqrt{\alpha} \omega_{0}$, $\left\langle x_{i}\right\rangle$ will be a positive quantity, because, while $\eta$ remains to be positive, the value of $h$ turns out to be negative in this case. Since the lattice displacement $x_{i}$ in the positive side makes $V\left(x_{i}\right)$ decrease from the corresponding value in the harmonic model due to the combined effects of the cubic and the quartic terms, we may expect that both anharmonicities shift $U_{\text {eff }}$ to the negative side, leading to another conclusion that the effect of the cubic anharmonicity depends critically on the sign of $g$. As shown in Figures 6(a)-6(d) for $\left\langle x_{i}\right\rangle, t_{\text {eff }}$, $U_{\text {eff, }}$ and $L_{0}$, we can clearly see the asymmetry with respect to $g$. For positive $g,\left\langle x_{i}\right\rangle$ deviates from the value in the harmonic case $-\sqrt{2 \alpha}$ (the dotted-dashed straight line) to a smaller magnitude of the lattice displacement. This change is associated with the increase in $U_{\text {eff, }} t_{\text {eff, }}$, and $L_{0}$. It may be noted that the increase in $t_{\text {eff }}$ has a direct consequence on the reduction of the optimized value for $\eta$ from the corresponding value in the harmonic system. Thus we may conclude that anharmonicity reduces the effective mass of a polaron in this case. On the other hand, $\left\langle x_{i}\right\rangle$ becomes much larger from the corresponding value in the harmonic case for negative $g$. In particular, $\left\langle x_{i}\right\rangle$ shows a very steep upturn 
if both $|g|$ and $\lambda_{3}$ are sufficiently large. This abrupt upturn is connected with the sudden change of the polaron feature into immobile particles due to the strong electron-electron attractive interaction. Judging from the fact that $L_{0}$ tends to vanish at the same time, we may further conclude that this change is nothing but the formation of immobile bipolarons.

In order to study the effect of anharmonicity on the phase transition, we have plotted $t_{\text {eff }} / t, d t_{\text {eff }} / d U$, and $L_{0}$ as a function of $U$ in Figures $7(\mathrm{a})-7(\mathrm{c})$, together with the energy per site, $\epsilon_{0}$ in Figure 8, respectively. For positive $g$, all the features obtained in these figures are qualitatively the same as those in the harmonic system (the dotted-dashed curves). Two peaks in $d t_{\text {eff }} / d U$ in Figure 7(b) along with $L_{0}=3 / 8$ for the state between the peaks clearly indicate the existence of an intervening metallic phase, leading to the observation that exactly the same phase transition is expected even in the anharmonic system. The quantitative difference can be accounted for by adjusting the values of both $U$ and $t$. Thus we may conclude that anharmonicity does not change the situation radically in this case. Only quantitative changes such as the reduction in the effective mass associated with the polaron motion occur. For negative $g$, however, we can see a similar behaviour only in the case of weak anharmonicity. For sufficiently strong $\lambda_{3}$ (but still within the realistic range), the feature of the transition changes radically. The intervening metallic state is suppressed and a direct firstorder SDW-to-CDW transition is predicted. The CDW is characterized by the formation of immobile bipolarons. This insulator-to-insulator transition is clearly seen by both the cusp in $\epsilon_{0}$ in Figure 8 and the jump in $L_{0}$ in Figure $7(\mathrm{c})$ from the vanishingly small value to the nearly full electron spin moment.

In the first-principles approach, $g$ is connected with $-\xi \hat{e} . \nabla V(\mathbf{r})$ in the rigid-ion approximation, where $V$ is the electron-ion interaction potential, $\xi$ is the time-dependent lattice displacement, and $\hat{e}$ is a polarization vector of the relevant lattice displacement. Since there is no definite rule for the direction of $\hat{e}$ and $V(\mathbf{r})$ may be considered as a pseudopotential, no definite argument can be made on the sign of $g$. Thus both positive and negative $g$ 's will appear in actual situations. From the viewpoint of superconductivity, we need at least a metallic phase. Thus the strong anharmonic case with negative $g$ should be avoided. However, anharmonicity with positive $g$ seems to provide a more favorable condition to support superconductivity, partly because the metallic region is widened and partly because the polarons become more mobile.

\section{An Improved Variational Treatment}

More recently, Clay and Hardikar [65] have studied the same model by a numerical method based on the densitymatrix renormalization group technique and confirmed the findings of TC. By calculating the Luttinger liquid correlation exponent $K_{q}$, they have further suggested that this phase is indeed superconductive. We believe that this issue is of paramount importance in the current scenario of superconductivity and needs a more rigorous analytical investigation. In a recent investigation we have chosen a more improved variational wave function for the phonon subsystem in order to obtain a lower GS energy and then analyzed the behaviour of the intermediate metallic phase. The idea has been that if the width of the metallic phase gets reduced in the improved calculation, then there can be an element of doubt about the veracity of the existence of the metallic phase. On the other hand, if an improved variational calculation leads to a widening of the metallic phase, then the prediction about the existence of the metallic phase becomes certainly much more justified.

In Section 3 we have described the method adopted by TC for the approximate solution of the phonon subsystem. TC have completely neglected in their analysis the phonon correlation effect which may play a rather important role as is well known from polaron physics. In the language of field theory, an electron is the source of phonons, and when an electron emits a phonon, it recoils back due to the finite phonon momentum, and while recoiling the electron can emit another phonon, particularly in the case of reasonable electron-phonon coupling, and in that case those two successively emitted virtual phonons will be correlated. This correlation leads to the squeezing of the coherent phonon state and it has been shown by Zheng [66] that it also reduces the Holstein reduction factor considerably and consequently makes the polaron bandwidth larger leading to a higher mobility of the polarons which is more favorable for high $T_{c}$ superconductivity. Therefore it is desirable that one should include the phonon correlations in the calculation. We have included the on-site (OS) and nearestneighbor $(\mathrm{NN})$ phonon correlations in one of our recent investigations. We have, however, neglected the anharmonic phonons in our calculation for the sake of simplicity. In fact, the inclusion of phonon correlations is expected to take care of the phonon anharmonicity at least partially. So we consider the same Hamiltonian (1) again. As before we perform first the transformation $e^{R_{1}}$ and $e^{R_{2}}$ where $R_{1}$ and $R_{2}$ are given by (2) and (7). As already mentioned earlier, we next perform, following Zheng [66], a squeezing transformation with a generator

$$
R_{3}=\alpha_{s} \sum_{i}\left(b_{i} b_{i}-b_{i}^{\dagger} b_{i}^{\dagger}\right),
$$

where $\alpha_{s}$ is the squeezing parameter to be obtained variationally. This transformation takes care of the phonon correlation at a particular site, and thus partially includes the electron recoil effect, and secondly, it takes into account through the choice of the phonon state some effects of the anharmonic phonons, that is, the phonon-phonon interactions which indirectly introduce the finite lifetime effects of the phonon and thus incorporates the phonon dynamics in a more realistic way. However, it neglects all intersite phonon correlations. Following Lo and Sollie [67], we, therefore, perform a correlated squeezing transformation with a generator

$$
R_{4}=\frac{1}{2} \sum_{i \neq j} \beta_{i j}\left(b_{i} b_{j}-b_{i}^{\dagger} b_{j}^{\dagger}\right),
$$




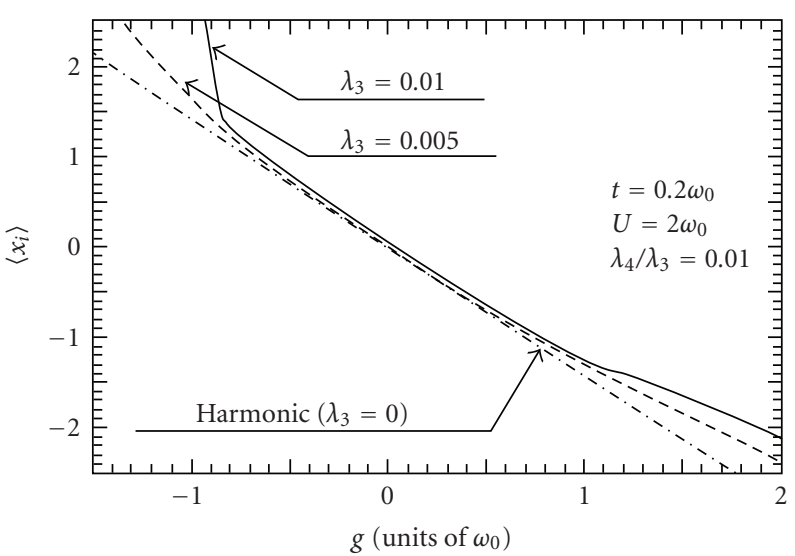

(a)

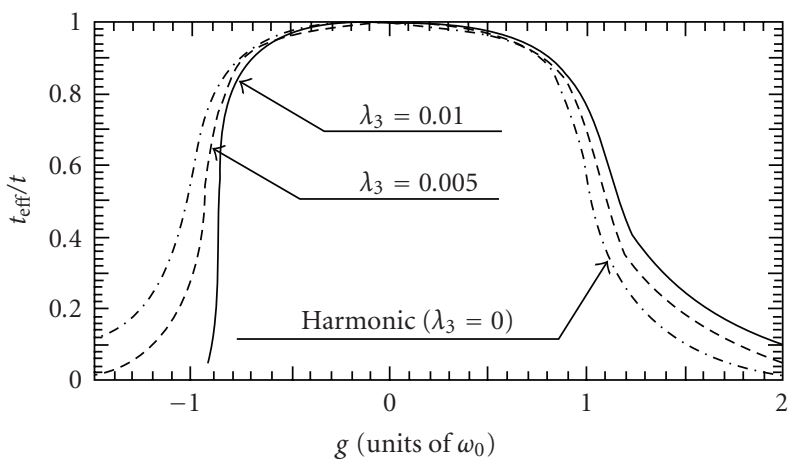

(c)

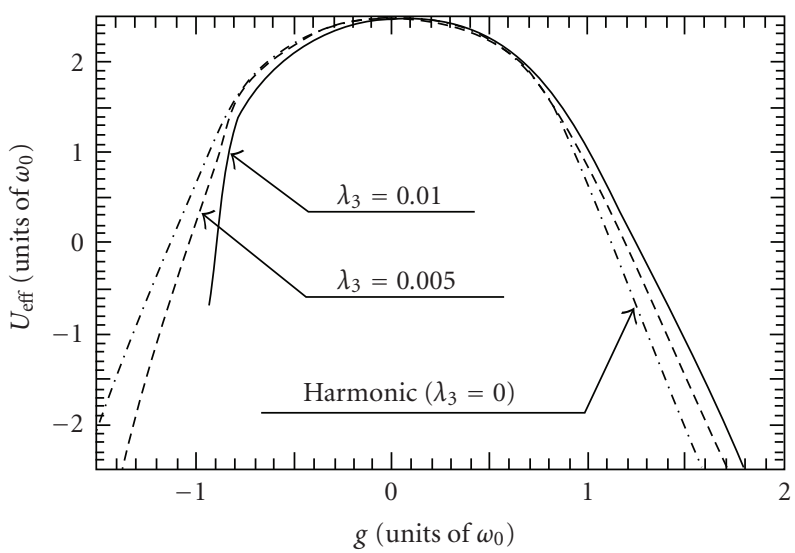

(b)

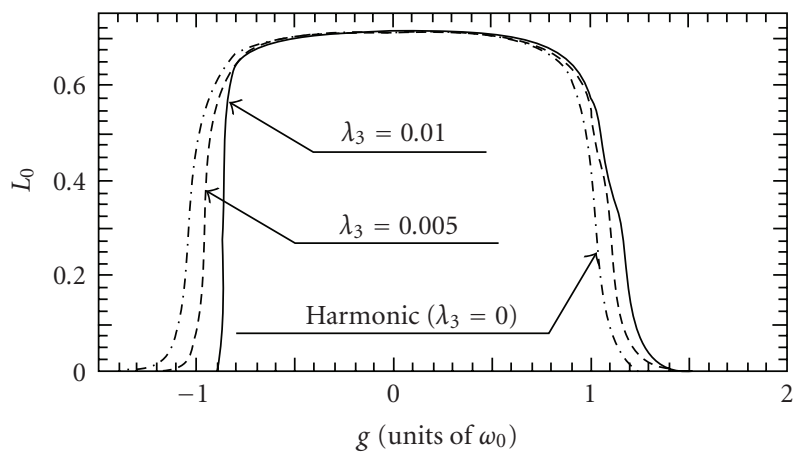

(d)

Figure 6: Plots of (a) the lattice displacement, (b) the effective electron-electron interaction, (c) the renormalized hopping integral, and (d) the local spin moment as a function of $g$.

where we choose, for simplicity, $\beta_{i j}=\beta$, when $i$ and $j$ are nearest neighbors and zero otherwise. Obviously the last two squeezing transformations (29) and (30) may spoil the coherence of phonons at the expense of including correlations and introduce some fluctuations. Therefore, in order to deal with these fluctuations, we perform another CST to bring back the complete phonon coherence with a generator

$$
R_{5}=\Delta \sum_{i}\left(b_{i}^{\dagger}-b_{i}\right),
$$

where $\Delta$ is another variational parameter. Averaging the transformed Hamiltonian with respect to the zero-phonon state $|0\rangle=\prod_{i}\left|0_{i}\right\rangle$, where the product over $i$ runs over $N$ sites, we then obtain an effective electronic Hamiltonian

$$
\begin{aligned}
H_{\mathrm{eff}}= & -J \sum_{i \sigma} n_{i \sigma}-t_{\mathrm{eff}} \sum_{\langle i j\rangle_{\sigma}} c_{i \sigma}^{\dagger} c_{j \sigma}+U_{\mathrm{eff}} \sum_{i} n_{i \uparrow} n_{i \downarrow} \\
& +\frac{N \omega_{0}\left[e^{4 \alpha}\left(e^{2 \beta}\right)_{00}+e^{-4 \alpha}\left(e^{-2 \beta}\right)_{00}-2\right]}{4} \\
& +N \omega_{0} h^{2}+N \omega_{0} M \Delta \mathrm{e}^{2 \alpha_{s}}\left(2 h+\Delta e^{2 \alpha_{s}}\right),
\end{aligned}
$$

with

$$
\begin{gathered}
J=\omega_{0}\left[\alpha(2-\eta)+2 \sqrt{\alpha}(1-\eta)\left(h+M \Delta e^{2 \alpha_{s}}\right)\right], \\
U_{\text {eff }}=\left[U-2 \alpha \omega_{0} \eta(2-\eta)\right], \\
t_{\text {eff }}=t \cdot \exp \left[\alpha \eta^{2} e^{-4 \alpha_{s}}\left(\left(e^{-2 \beta}\right)_{00}-\left(e^{-2 \beta}\right)_{01}\right)\right], \\
M=\left(e^{\beta}\right)_{00}+2\left[\left(e^{\beta}\right)_{01}+\left(e^{\beta}\right)_{02}+\left(e^{\beta}\right)_{03}+\cdots\right], \\
\left(e^{ \pm 2 \beta}\right)_{0 n}=\sum_{m=0,1,2, \ldots}( \pm 1)^{n} \frac{(2 \beta)^{2 m+n}}{m !(m+n) !} .
\end{gathered}
$$

The GS energy per site is, thus, obtained for all values of $U$ (both positive and negative) as

$$
\begin{aligned}
\varepsilon_{0}= & -J+N \omega_{0}\left[h^{2}+\frac{\left(\left(e^{2 \beta}\right)_{00} \cosh 4 \alpha-1\right)}{2}\right] \\
& +\left[\frac{\left(U_{\text {eff }}-\left|U_{\text {eff }}\right|\right)}{4}\right]+N \Delta \omega_{0} M e^{2 \alpha_{s}}\left(2 h+\Delta e^{2 \alpha_{s}}\right) \\
& -4 t_{\text {eff }} \int_{0}^{\infty} d y \frac{J_{0}(y) J_{1}(y)}{y\left[1+\exp \left(y\left|U_{\text {eff }}\right| / 2 t_{\text {eff }}\right)\right]} .
\end{aligned}
$$

The GS energy is now minimized with respect to five variational parameters. It is found that the energy results 


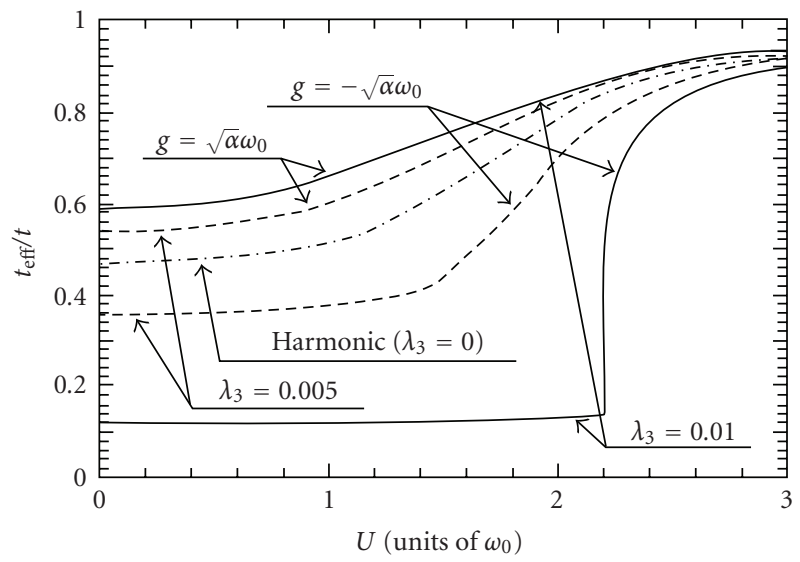

(a)

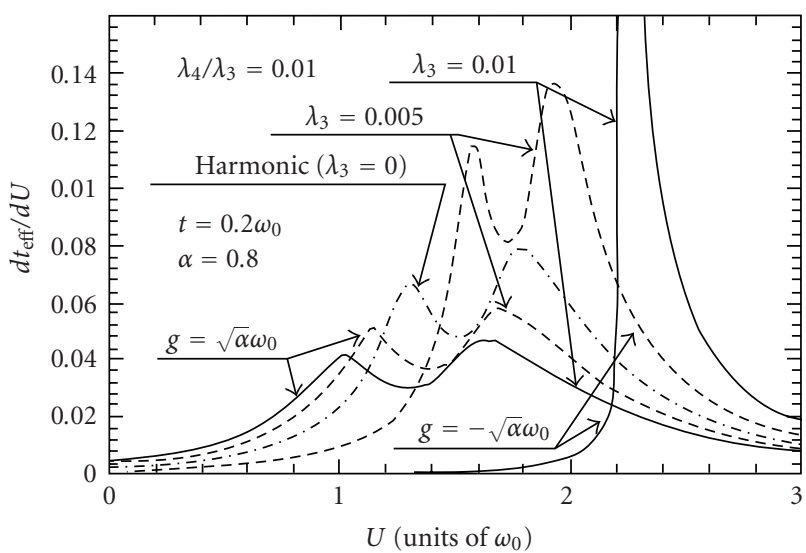

(b)

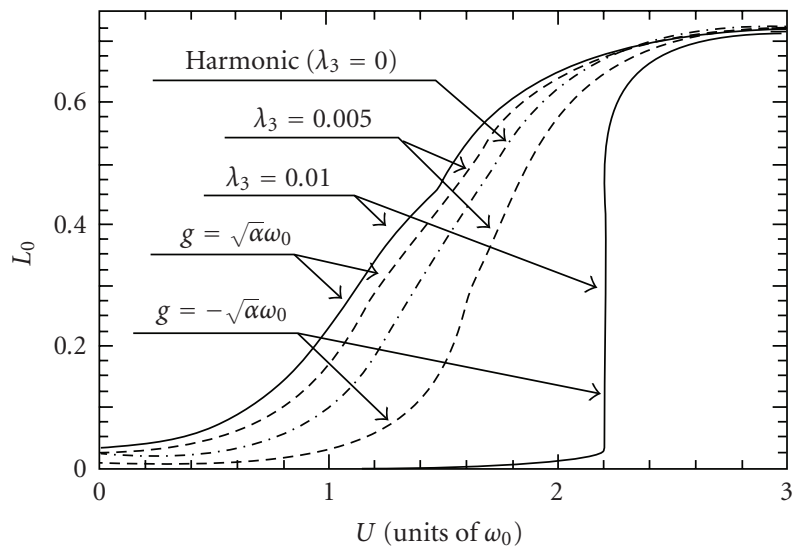

(c)

FIGURE 7: Plots of (a) the renormalized hopping integral, (b) the derivative of the renormalized hopping integral with respect to $U$, and (c) the local spin moment as a function of $U$.

are only marginally better. However, we find that even this marginal improvement in the energy can have substantial effect on the polaron mobility and the phase diagram.

One can see from Figure 9 that $t_{\text {eff }}$ and its derivative show qualitatively the same behaviour as obtained by TC, but the present solutions show pronounced quantitative difference,

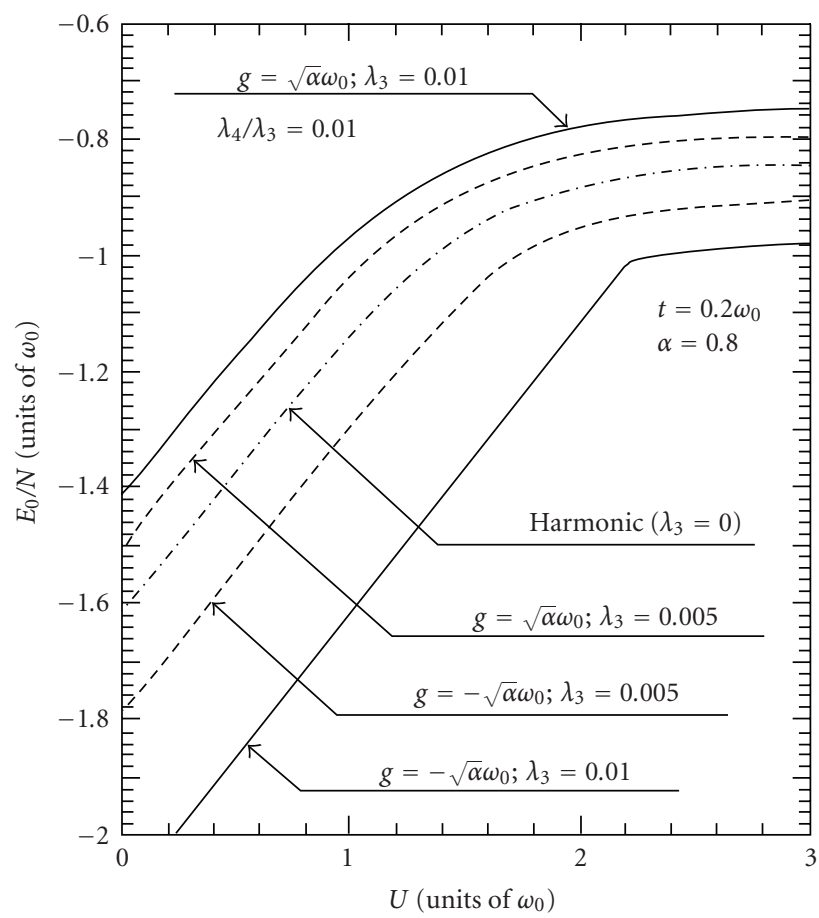

Figure 8: Ground-state energy per site as a function of $U$.

as clearly evident from the peak structures in the $d t_{\text {eff }} / d U$ curves (Figure 9(b)). The important point to note here is that the present method leads to an enhancement in the polaron mobility as compared with the TC results save for a small region of $U$ where the two methods almost agree. Again, the region in between the transition points satisfies the criterion $t_{\text {eff }} / U_{\text {eff }} \gg 0.25$, typical of a metallic state. Now the peaks are a little wider apart as compared to the TC results. Figure 10 shows the phase diagram in the parameter space $(\alpha, U)$ and comparison with the TC results again clearly shows that the present method predicts a wider metallic phase at the crossover region. Including the phonon anharmonicity at the Hamiltonian level will make the intermediate metallic phase even more wider, as shown in [45] and already discussed in Section 5, and the polarons more mobile, which is certainly a more favorable situation for high- $T_{c}$ superconductivity.

\section{Conclusion}

We have first considered the Holstein-Hubbard model at half-filling in one dimension and used a variable Lang-Firsov transformation and coherent state transformation with a generator which is independent of the electron variables. The transformed Hamiltonian is then averaged with respect to the unperturbed zero-phonon state to obtain an effective electronic Hamiltonian which could be solved exactly using the Bethe ansatz method of Lieb and Wu. Calculation of the polaron bandwidth and the local spin density have shown that there can exist an intermediate metallic state at the crossover region of SDW and CDW phases. It is not known for sure whether this kind of a metallic phase has already been observed in actual materials or not, but Takada has 


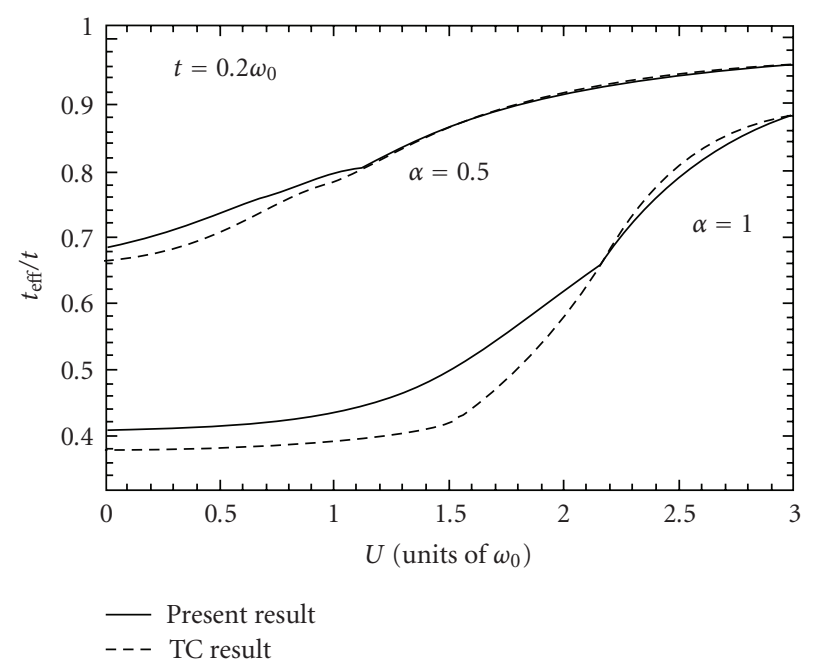

(a)

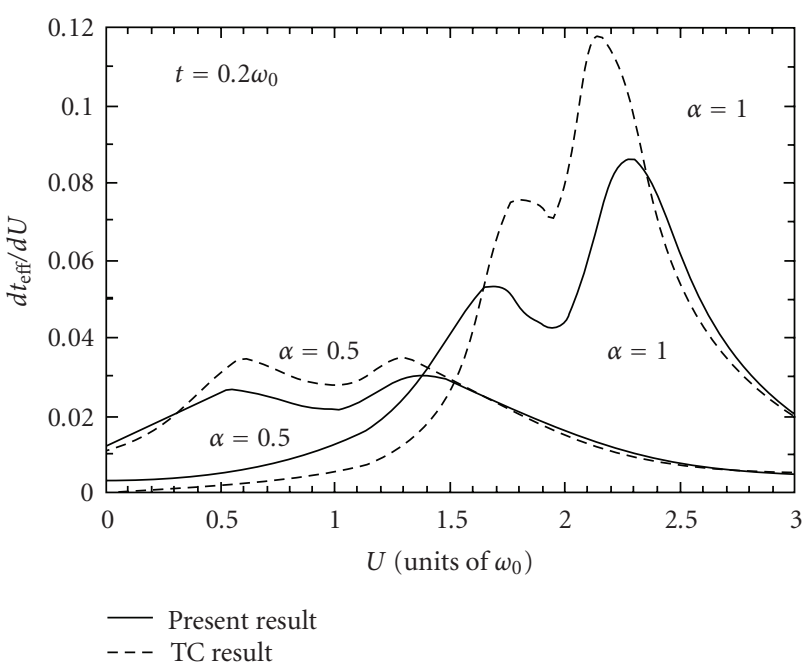

(b)

FIGURE 9: $t_{\mathrm{eff}}$ and its derivative as a function of $U$ for two values of $\alpha$ and for $t=0.2 \omega_{0}$. The TC results are shown for comparison.

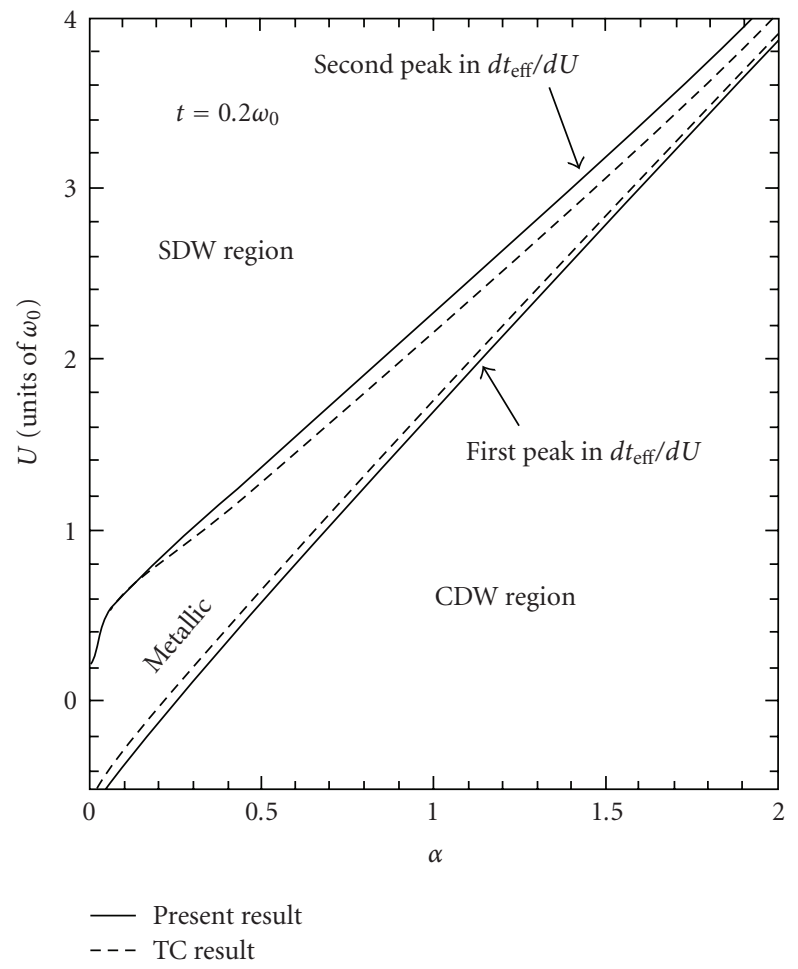

Figure 10: Phase diagram in $(\alpha, U)$ determined by the peaks in $d t_{\text {eff }} / d U$. The corresponding phase diagram obtained by TC in [44] is shown in dashed lines for comparison.

already speculated that the physical properties including superconductivity in the alkali-doped fullerenes $\left(A_{3} C_{60}\right)[68$, 69] can be explained in a unified way in terms of a picture that envisages the existence of such a kind of a metallic state in $\left(A_{3} C_{60}\right)[70,71]$. This metallic state is of importance from the point of view of superconductivity. We have next shown that the inclusion of phonon anharmonicity can make polarons more mobile and widen the metallic regime and can, thus, provide a more favorable condition to support superconductivity. Our prediction has been supported by a recent exact DMRG analysis. We have finally attempted to make a further improvement on our variational calculation by including the effect of phonon correlations through onsite and correlated squeezing transformations. Interestingly, this widens the metallic phase even further and makes the polarons more mobile which is more conducive for superconductivity. That a better variational calculation does widen the metallic phase is itself a positive test for its existence. We do not believe that such a behaviour is unique in 1D and we expect a similar behaviour in higher dimensions too. We do not believe that the case in point is a usual phase transition of the thermodynamic kind; rather it could be a quantum phase transition or a crossover phenomenon. It is of course possible to explore the superconducting nature of this phase within the present scenario. In fact, it goes without saying that being a metallic phase, this phase will certainly be superconductive as one will cool the temperature. However to find the transition temperature itself, one does need to do a rigorous calculation. It is, however, worthwhile to make a few qualitative remarks about the nature of the superconducting phase. The normal phase here is a polaronic or a bipolaronic metal. Therefore as the temperature would decrease, the system can undergo a polaronic superconductivity induced by dynamical pairing of polarons like what happens in the case of Cooper pairs in the BCS mechanism. On the other hand, it is also possible that bipolarons which are essentially "static" Cooper pairs can undergo Bose-Einstein condensation and give rise to a superconductive phase. Which route will actually lead to the superconductive phase in an actual system will depend on the characteristic temperatures of the corresponding mechanisms which will certainly depend on the material parameters, and therefore requires a rigorous calculation. Another remark that we would like to make is about 
the assumption of half-filling in the present analysis. This assumption has been used in order to obtain analytical results. Even for the case of "away from half-filling" exact result can be obtained for the effective Hubbard model, albeit numerically. For less than half-filling, the system will have more mobility in the charge carriers, and therefore it is expected that the metallic phase will be more favorable in this case than that for half-filling. For more than half-filling, the situation is not very clear and one needs to make a separate investigation.

\section{References}

[1] J. G. Bednorz and K. A. Müller, "Possible high $T_{c}$ superconductivity in the $\mathrm{Ba}-\mathrm{La}-\mathrm{Cu}-\mathrm{O}$ system," Zeitschrift für Physik B, vol. 64, no. 2, pp. 189-193, 1986.

[2] J. W. Halley, Ed., Theories of High Temperature Superconductivity, Addison Wesley, Harlow, UK, 1988.

[3] T. Jarlborg, "Band structure and electron-phonon coupling in the $\mathrm{La}_{1-x} \mathrm{SrCuO}$ superconductors," Helvetica Physica Acta, vol. 61, p. 421, 1988.

[4] R. E. Cohen, W. E. Pickett, H. Krakauer, and D. A. Papaconstan-topoulos, "High $T_{c}$ superconductors as ionic metals and the role of phonons in high $T_{c}$ superconductivity," Phase Transitions, vol. 22, no. 1-4, pp. 167-183, 1990.

[5] R. E. Cohen, W. E. Pickett, and H. Krakauer, "Theoretical determination of strong electron-phonon coupling in $\mathrm{YBa}_{2} \mathrm{Cu}_{3} \mathrm{O}_{7}$," Physical Review Letters, vol. 64, no. 21, pp. 25752578, 1990.

[6] C. O. Rodriguez, A. I. Liechtenstein, I. I. Mazin, O. Jepsen, O. K. Andersen, and M. Methfessol, "Optical near-zone-center phonons and their interaction with electrons in $\mathrm{YBa}_{2} \mathrm{Cu}_{3} \mathrm{O}_{7}$ : results of the local-density approximation," Physical Review B, vol. 42, no. 4, pp. 2692-2695, 1990.

[7] R. Zeyher, "Improtance of long-range electron-phonon coupling in high- $T_{c}$ superconductors," Zeitschrift für Physik B, vol. 80, no. 2, pp. 187-192, 1990.

[8] B. K. Chakraverty, D. Feinberg, Z. Hang, and M. Avignon, "Squeezed bipolaronic states and high temperature superconductivity in BaLaCuO systems," Solid State Communications, vol. 64, no. 8, pp. 1147-1151, 1987.

[9] D. Emin, "Formation, motion, and high-temperature superconductivity of large bipolarons," Physical Review Letters, vol. 62, no. 13, pp. 1544-1547, 1989.

[10] D. Emin and M. S. Hillery, "Formation of a large singlet bipolaron: application to high-temperature bipolaronic superconductivity," Physical Review B, vol. 39, no. 10, pp. 65756593, 1989.

[11] J. Ranninger, in Proceedings of the Conference on Lattice Effects in High Tc Superconductors, Y. Bar-Yam, T. Egami, J. Mustre de Leon, and A. R. Bishop, Eds., p. 389, 1992.

[12] S. Sil and A. Chatterjee, "Multi-dimensional Fröhlich bipolaron and dimensional scaling," International Journal of Modern Physics B, vol. 4, no. 11-12, pp. 1879-1888, 1990.

[13] S. Sil and A. Chatterjee, "Stability of large optical singlet bipolarons, many-particle effects and high temperature superconductivity," Modern Physics Letters B, vol. 6, no. 15, pp. 959966, 1992.

[14] J. R. Hardy and J. W. Hocken, "Possible origins of high- $T_{c}$ superconductivity," Physical Review Letters, vol. 60, no. 21, pp. 2191-2193, 1988.
[15] A. S. Alexandrov and N. F. Mott, "Bipolarons," Reports on Progress in Physics, vol. 57, no. 12, pp. 1197-1288, 1994.

[16] R. Micnas, J. Ranninger, and S. Robaszkiewicz, "Superconductivity in narrow-band systems with local nonretarded attractive interactions," Reviews of Modern Physics, vol. 62, no. 1, pp. 113-171, 1990.

[17] A. J. Mills, P. B. Littlewood, and B. I. Shraiman, "Double exchange alone does not explain the resistivity of $\mathrm{La}_{1-x} \mathrm{Sr}_{x} \mathrm{MnO}_{3}$," Physical Review Letters, vol. 74, no. 25, pp. 5144-5147, 1995.

[18] K. H. Kim, J. Y. Gu, H. S. Choi, G. W. Park, and T. W. Noh, "Frequency shifts of the internal phonon modes in $\mathrm{La}_{0.7} \mathrm{Ca}_{0.3} \mathrm{Mno}_{3}$," Physical Review Letters, vol. 77, no. 9, pp. 1877-1880, 1996.

[19] R. P. Sharma, L. E. Rehn, P. M. Baldo, and J. Z. Liu, "Direct evidence of anomalous $\mathrm{Cu}-\mathrm{O}$ vibrational modes near $T_{c}$ in $\mathrm{ErBa}_{2} \mathrm{Cu}_{3} \mathrm{O}_{7-\delta}$," Physical Review Letters, vol. 62, no. 24, pp. 2869-2872, 1989.

[20] R. P. Sharma, L. E. Rehn, P. M. Baldo, and J. Z. Liu, "Shift of phonon anomaly with $T_{c}$ observed in (Y,Er) $\mathrm{Ba}_{2} \mathrm{Cu}_{3} \mathrm{O}_{7-\delta}$ - by ion channeling," Physical Review B, vol. 40, no. 16, pp. 1139611399, 1989.

[21] M. Arai, K. Yamada, Y. Hidaka, et al., "Anomaly of phonon state of superconducting $\mathrm{YBa}_{2} \mathrm{Cu}_{3} \mathrm{O}_{7}$ studied by inelastic neutron scattering," Physical Review Letters, vol. 69, no. 2, pp. 359-362, 1992.

[22] C. M. Foster, A. J. Heeger, Y. H. Kim, G. Stucky, and N. Herron, "Photogenerated carriers in $\mathrm{La}_{2} \mathrm{CuO}_{4}, \mathrm{YBa}_{2} \mathrm{Cu}_{3} \mathrm{O}_{7-\delta}$ and $\mathrm{TI}_{2} \mathrm{Ba}_{2} \mathrm{Ca}_{(1-x)} \mathrm{Gd}_{x} \mathrm{Cu}_{2} \mathrm{O}_{8}$ : polarizability-induced pairing of polarons," Synthetic Metals, vol. 33, no. 2, pp. 171-183, 1989.

[23] D. Mihailović, C. M. Foster, K. Voss, and A. J. Heeger, "Application of the polaron-transport theory to $\sigma(\omega)$ in $\mathrm{TI}_{2} \mathrm{Ba}_{2} \mathrm{Ca}_{1-x} \mathrm{Gd}_{x} \mathrm{Cu}_{2} \mathrm{O}_{8}, \mathrm{YBa}_{2} \mathrm{Cu}_{3} \mathrm{O}_{7-\delta}$, and $\mathrm{La}_{2-x} \mathrm{Sr}_{x} \mathrm{CuO}_{4}$," Physical Review B, vol. 42, no. 13, pp. 7989-7993, 1990.

[24] S. D. Conradson, I. D. Raistrick, and A. R. Bishop, "Axial oxygen-centered lattice instabilities and high-temperature superconductivity," Science, vol. 248, no. 4961, pp. 1394-1398, 1990.

[25] J. Mustre de Leon, S. D. Conradson, I. Batistic, and A. R. Bishop, "Evidence for an axial oxygen-centered lattice fluctuation associated with the superconducting transition in $\mathrm{YBa}_{2} \mathrm{Cu}_{3} \mathrm{O}_{7}$," Physical Review Letters, vol. 65, no. 13, pp. 1675$1678,1990$.

[26] H. A. Mook, M. Mostoller, J. A. Harvey, N. W. Hill, B. C. Chakoumakos, and B. C. Sales, "Observation of phonon softening at the superconducting transition in $\mathrm{Bi}_{2} \mathrm{Sr}_{2} \mathrm{CaCu}_{2} \mathrm{O}_{8}$," Physical Review Letters, vol. 65, no. 21, pp. 2712-2715, 1990.

[27] H. A. Mook, B. C. Chakoumakos, M. Mostoller, A. T. Boothroyd, and D. M. Paul, "Phonons and superconductivity in $\mathrm{Bi}_{2} \mathrm{Sr}_{2} \mathrm{CaCu}_{2} \mathrm{O}_{8}$," Physical Review Letters, vol. 69, no. 15, pp. 2272-2275, 1992.

[28] T. Holstein, "Studies of polaron motion-part I: the molecular-crystal model," Annals of Physics, vol. 8, no. 3, pp. 325-342, 1959.

[29] T. K. Mitra, A. Chatterjee, and S. Mukhopadhyay, "Polarons," Physics Reports, vol. 153, no. 2-3, pp. 91-207, 1987.

[30] J. Bardeen, L. N. Cooper, and J. R. Schrieffer, "Theory of superconductivity," Physical Review, vol. 108, no. 5, pp. 11751204, 1957.

[31] A. Alexandrov and J. Ranninger, "Theory of bipolarons and bipolaronic bands," Physical Review B, vol. 23, no. 4, pp. 17961801, 1981. 
[32] A. N. Das and S. Sil, "Electron-phonon interaction in a strongly correlated Hubbard system," Physica C, vol. 161, no. 3, pp. 325-330, 1989.

[33] E. Berger, P. Valaśěk, and W. von der Linden, "Twodimensional Hubbard-Holstein model," Physical Review B, vol. 52, no. 7, pp. 4806-4814, 1995.

[34] S. Sil and B. Bhattacharyya, "Effect of electron-phonon interaction on a one-dimensional correlated electron system," Physical Review B, vol. 54, no. 20, pp. 14349-14354, 1996.

[35] C.-H. Pao and H.-B. Schüttler, "Superconducting instability in the Holstein-Hubbard model: a numerical renormalizationgroup study," Physical Review B, vol. 57, no. 9, pp. 5051-5054, 1998.

[36] A. Weiße, H. Fehske, G. Wellein, and A. R. Bishop, "Optimized phonon approach for the diagonalization of electron-phonon problems," Physical Review B, vol. 62, no. 2, pp. R747-R750, 2000.

[37] V. Cataudella, G. De Filippis, and G. Iadonisi, "Polaron features of the one-dimensional Holstein molecular crystal model," Physical Review B, vol. 62, no. 3, pp. 1496-1499, 2000.

[38] W. Koller, A. C. Hewson, and D. M. Edwards, "Polaronic quasiparticles in a strongly correlated electron band," Physical Review Letters, vol. 95, no. 25, Article ID 256401, 4 pages, 2005.

[39] G. Sangiovanni, M. Capone, C. Castellani, and M. Grilli, "Electron-phonon interaction close to a mott transition," Physical Review Letters, vol. 94, no. 2, Article ID 026401, 4 pages, 2005.

[40] P. Barone, R. Raimondi, M. Capone, and C. Castellani, "Effective electron-phonon coupling and polaronic transition in the presence of strong correlation," Physical Review B, vol. 73, no. 8, Article ID 085120, 5 pages, 2006.

[41] Y. Takada, "Superconductivity in the half-filled HubbardHolstein model in the antiadiabatic region," Journal of the Physical Society of Japan, vol. 65, no. 6, pp. 1544-1547, 1996.

[42] E. Jeckelmann, C. Zhang, and S. R. White, "Metal-insulator transition in the one-dimensional Holstein model at half filling," Physical Review B, vol. 60, no. 11, pp. 7950-7955, 1999.

[43] Q. Wang, H. Zheng, and M. Avignon, "Phase diagram and optical conductivity of the one-dimensional spinless Holstein model," Physical Review B, vol. 63, no. 1, Article ID 014305, 5 pages, 2001.

[44] Y. Takada and A. Chatterjee, "Possibility of a metallic phase in the charge-density-wave-spin-density-wave crossover region in the one-dimensional Hubbard-Holstein model at half filling," Physical Review B, vol. 67, no. 8, Article ID 081102, 4 pages, 2003.

[45] A. Chatterjee and Y. Takada, "The Hubbard-Holstein model with anharmonic phonons in one dimension," Journal of the Physical Society of Japan, vol. 73, no. 4, pp. 964-969, 2004.

[46] P. M. Krishna and A. Chatterjee, "Existence of a metallic phase in a 1D Holstein-Hubbard model at half filling," Physica C, vol. 457, no. 1-2, pp. 55-59, 2007.

[47] E. H. Lieb and F. Y. Wu, "Absence of mott transition in an exact solution of the short-range, one-band model in one dimension," Physical Review Letters, vol. 20, no. 25, pp. 1445$1448,1968$.

[48] I. G. Lang and Yu. A. Firsov, Zh. Éksp. Teor. Fiz., vol. 43, p. 1843, 1962, Soviet Physics—JETP, vol. 16, p. 1301, 1963.

[49] A. N. Das and S. Sil, "A study of the polaronic band width and the small-to-large-polaron transition in a many-polaron system," Journal of Physics: Condensed Matter, vol. 5, no. 44, pp. 8265-8276, 1993.
[50] M. Zoli and A. N. Das, "Polaron crossover in molecular solids," Journal of Physics: Condensed Matter, vol. 16, no. 21, pp. 35973607, 2004.

[51] M. Frick, I. Morgenstern, and W. von der Linden, "Hightemperature superconductivity in the apex-oxygen model: a quantum Monte Carlo study," Zeitschrift für Physik B, vol. 82, no. 3, pp. 339-345, 1991.

[52] M. Frick, I. Morgenstern, and W. von der Linden, "Anharmonic phonons and strong electronic correlations in high- $T_{c}$ superconductors: a Quantum Monte Carlo study," Physica $C$, vol. 185-189, part 3, pp. 1523-1524, 1991.

[53] A. Bussmann-Holder and A. R. Bishop, "Anharmonicityinduced multiphonon processes in high-temperature superconductors," Physical Review B, vol. 44, no. 6, pp. 2853-2856, 1991.

[54] N. M. Plakida, V. L. Aksenov, and S. L. Drechsler, "Anharmonic model for high- $T_{c}$ superconductors," Europhysics Letters, vol. 4, no. 11, pp. 1309-1314, 1987.

[55] N. M. Plakida, "Lattice instability and strong electron-phonon coupling for high- $T_{c}$ superconductivity," Physica C, vol. 162164, part 2, pp. 1341-1342, 1989.

[56] N. M. Plakida and S. E. Krasavin, "A microscopical model of anharmonic lattice dynamics of $\mathrm{La}_{2} \mathrm{CuO}_{4}$," Physica $C$, vol. 185-189, part 3, pp. 1531-1532, 1991.

[57] H. de Raedt, T. Schneider, and M. P. Sörensen, "Superconductivity in Hubbard models coupled to non-fermionic degrees of freedom," Zeitschrift für Physik B, vol. 79, no. 3, pp. 327-332, 1990.

[58] J. Konior, "Anharmonic polaronic model and high- $T_{c}$ superconductivity," Physical Review B, vol. 47, no. 21, pp. 1442514433, 1993.

[59] J. K. Freericks and G. D. Mahan, "Strong-coupling expansions for the anharmonic Holstein model and for the HolsteinHubbard model," Physical Review B, vol. 54, no. 13, pp. 93729384, 1996.

[60] G. D. Mukherjee, C. Bansal, and A. Chatterjee, "Thermal expansion study of ordered and disordered $\mathrm{Fe}_{3} \mathrm{Al}$ : an effective approach for the determination of vibrational entropy," Physical Review Letters, vol. 76, no. 11, pp. 1876-1879, 1996.

[61] G. D. Mukherjee, C. Bansal, and A. Chatterjee, "Thermal expansion analysis of $\mathrm{Fe}_{3-x} \mathrm{Mn}_{x} \mathrm{Al}$ alloys," Physical Review B, vol. 58, no. 10, pp. 6172-6179, 1998.

[62] G. D. Mukherjee, A. Chatterjee, and C. Bansal, "Anomalous thermal expansion behavior of the $\mathrm{YBaCuO}$ superconductor. Indirect evidence of polaron formation," Physica C, vol. 232, no. 3-4, pp. 241-245, 1994.

[63] G. D. Mukherjee, C. Bansal, and A. Chatterjee, "Thermal expansion study of Bi-2223 superconductor: an evidence of polaronic mechanism of high $T_{c}$ superconductivity with strong two-dimensional fluctuations," Physica C, vol. 333, no. 3, pp. 229-234, 2000.

[64] C. Kittel, Introduction to Solid State Physics, John Wiley \& Sons, New York, NY, USA, 1996.

[65] R. T. Clay and R. P. Hardikar, "Intermediate phase of the one dimensional half-filled Hubbard-Holstein model," Physical Review Letters, vol. 95, no. 9, Article ID 096401, 4 pages, 2005.

[66] H. Zheng, "Squeezed polarons in one dimension," Physics Letters A, vol. 131, no. 2, pp. 115-118, 1988.

[67] C. F. Lo and R. Sollie, "Correlated squeezed polaron states in one dimension,” Physical Review B, vol. 48, no. 14, pp. 1018310187, 1993.

[68] A. F. Hebard, M. J. Rosseinsky, R. C. Haddon, et al., "Superconductivity at $18 \mathrm{~K}$ in potassium-doped C60," Nature, vol. 350, no. 6319, pp. 600-601, 1991. 
[69] O. Gunnarsson, "Superconductivity in fullerides," Reviews of Modern Physics, vol. 69, no. 2, pp. 575-606, 1997.

[70] Y. Takada, "Explanation of the anomalous isotope effect in superconducting alkali-metal-doped fullerenes," Journal of the Physical Society of Japan, vol. 65, no. 10, pp. 3134-3137, 1996.

[71] Y. Takada and T. Hotta, "Superconductivity in the alkali-doped fullerides: competition of phonon-mediated attractions with coulomb repulsions in polaron pairing," International Journal of Modern Physics B, vol. 12, no. 29-31, pp. 3042-3051, 1998. 

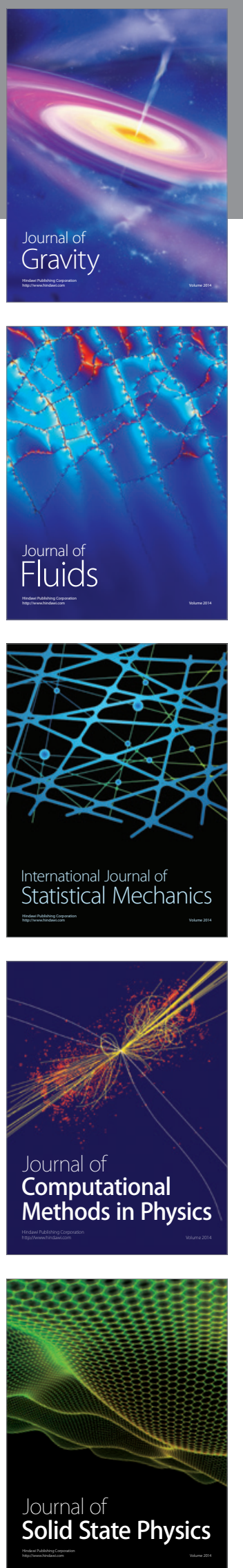

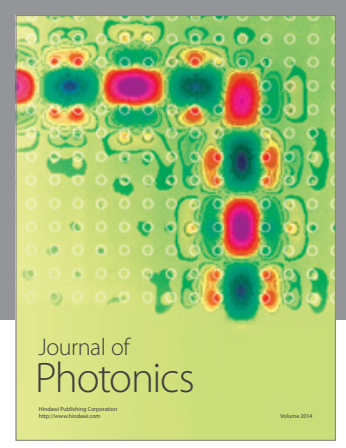

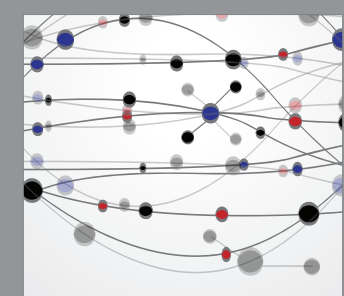

The Scientific World Journal
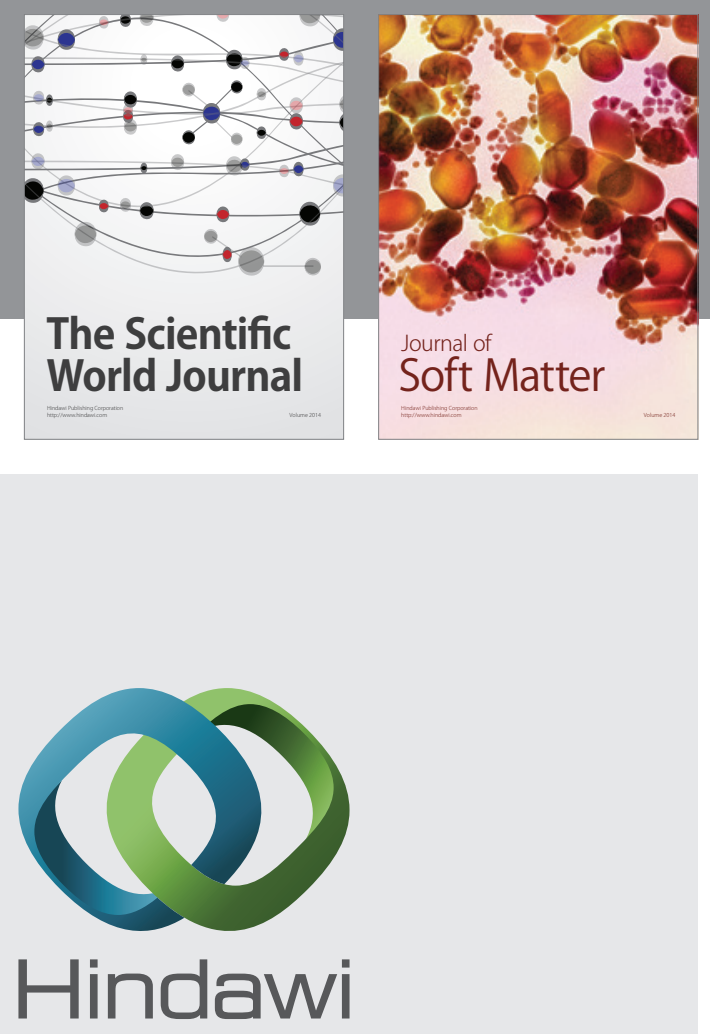

Submit your manuscripts at

http://www.hindawi.com
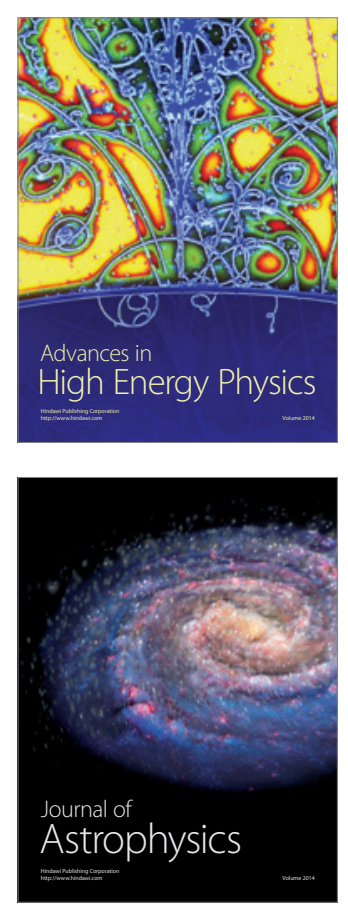
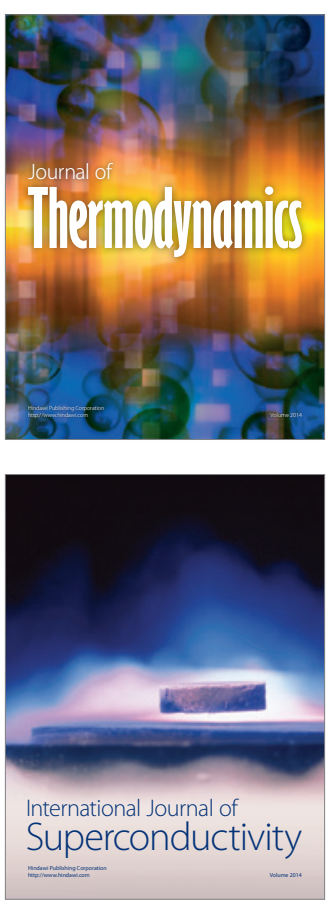
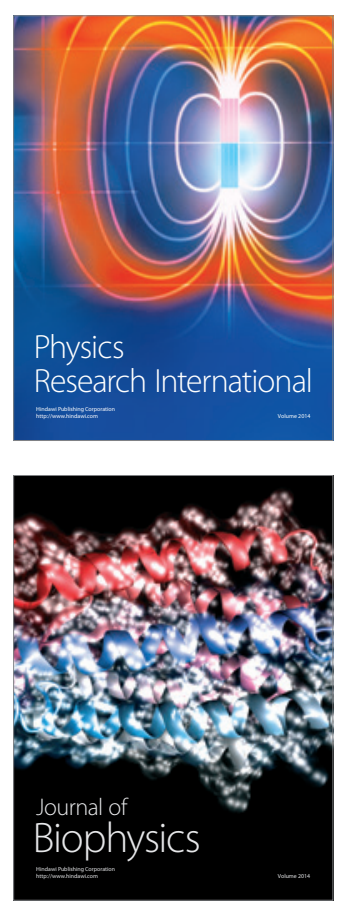
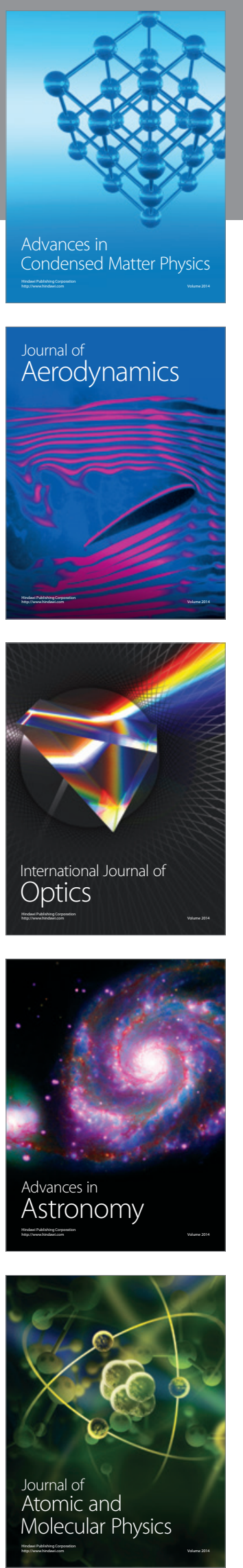